PASJ: Publ. Astron. Soc. Japan , 1-??,

(C) 2018. Astronomical Society of Japan.

\title{
Subaru FOCAS Spectroscopic Observations of High-Redshift Supernovae*
}

\author{
Tomoki Morokuma ${ }^{1,2}$, Kouichi Tokita ${ }^{3}$, Christopher Lidman ${ }^{4}$, Mamoru Doi ${ }^{3,5}$, Naoki YasudA ${ }^{5,6}$, Greg \\ Aldering $^{7}$, Rahman Amanullah ${ }^{7,8}$, Kyle Barbary ${ }^{7}$, Kyle Dawson ${ }^{9}$, Vitaliy FadeYev ${ }^{10}$, Hannah K. \\ Fakhouri $^{7}$, Gerson Goldhaber ${ }^{7}$, Ariel Goobar ${ }^{8}$, Takashi Hattori ${ }^{11}$, Junji HaYAno ${ }^{3}$, Isobel M. Hook ${ }^{12,13}$, D. \\ Andrew Howeld ${ }^{14,15}$, Hisanori Furusawa ${ }^{1}$, Yutaka Ihara ${ }^{2,3}$, Nobunari Kashikawa ${ }^{1}$, Rob A. Knop ${ }^{16}$, Kohki \\ Konishi $^{6}$, Joshua Meyers ${ }^{7}$, Takeshi Oda ${ }^{17}$, Reynald Pain ${ }^{18}$, Saul Perlmutter ${ }^{7}$, David Rubin ${ }^{7}$, Anthony L. \\ Spadafora $^{7}$, Nao Suzuki ${ }^{7}$, Naohiro TAkAnashi ${ }^{1}$, Tomonori Totani ${ }^{17}$, Hiroyuki Utsunomiya ${ }^{3}$, Lifan Wang $^{19}$ \\ (Supernova Cosmology Project) \\ ${ }^{1}$ National Astronomical Observatory of Japan, 2-21-1 Osawa, Mitaka, Tokyo 181-8588, Japan \\ ${ }^{2}$ Research Fellow of the Japan Society for the Promotion of Science \\ ${ }^{3}$ Institude of Astronomy, Graduate School of Science, University of Tokyo, 2-21-1, Osawa, Mitaka, Tokyo 181-0015, Japan \\ ${ }^{4}$ Oskar Klein Centre, Stockholm University, AlbaNova University Centre, 10691 Stockholm, Sweden \\ ${ }^{5}$ Institute of Physics and Mathematics of the Universe, University of Tokyo, Kashiwa, Chiba 27r7-8582, Japan \\ ${ }^{6}$ Institute for Cosmic Ray Research, University of Tokyo, Kashiwa, Chiba 277-8582, Japan \\ ${ }^{7}$ Lawrence Berkeley National Laboratory, 1 Cyclotron Road, Berkeley, CA 94720, USA \\ ${ }^{8}$ Physics Department, Stockholm University, AlbaNova University Centre, 10691 Stockholm, Sweden \\ ${ }^{9}$ Department of Physics and Astronomy, University of Utah, Salt Lake City, UT 84112, USA \\ ${ }^{10}$ Santa Cruz Institute for Particle Physics, University of Californica, Santa Cruz, CA 94064, USA \\ ${ }^{11}$ Subaru Telescope, National Astronomical Observatory of Japan, 650 North A'ohoku Place, Hilo, HI 96720, USA \\ ${ }^{12}$ University of Oxford Astrophysics, Denys Wilkinson Building, Keble Road, Oxford OX1 3RH, UK \\ ${ }^{13}$ INAF - Observatorio Astronomico di Roma, via Frascati 33, 00040, Monteporzio (RM), Italy \\ ${ }^{14}$ Las Cumbres Observatory Global Telescope Network, 6740 Cortona Dr., Suite 102, Goleta, CA 93117, USA \\ ${ }^{15}$ Department of Physics, University of California, Santa Barbara, Broida Hall, Mail Code 9530, Santa Barbara, CA \\ 93106-9530 \\ ${ }^{16}$ Department of Physics and Astronomy, Vanderbilt University, P.O. Box 1807, Nashville, TN 37240, USA \\ ${ }^{17}$ Department of Astronomy, Kyoto University, Sakyo-ku, Kyoto 606-8502, Japan \\ ${ }^{18}$ LPNHE, CNRS-IN2P3 and University of Paris VI and VII, 75005 Paris, France \\ ${ }^{19}$ Physics Department, Texas AEM University, College Station, TX 77843, USA \\ tomoki.morokuma@nao.ac.jp
}

(Received 2009 July 30; accepted 2009 November 6)

\begin{abstract}
We present spectra of high-redshift supernovae (SNe) that were taken with the Subaru low resolution optical spectrograph, FOCAS. These SNe were found in SN surveys with Suprime-Cam on Subaru, the CFH12k camera on the Canada-France-Hawaii Telescope (CFHT), and the Advanced Camera for Surveys (ACS) on the Hubble Space Telescope (HST). These SN surveys specifically targeted $z>1$ Type Ia supernovae (SNe Ia). From the spectra of 39 candidates, we obtain redshifts for 32 candidates and spectroscopically identify 7 active candidates as probable SNe Ia, including one at $z=1.35$, which is the most distant SN Ia to be spectroscopically confirmed with a ground-based telescope. An additional 4 candidates are identified as likely SNe Ia from the spectrophotometric properties of their host galaxies. Seven candidates are not SNe Ia, either being SNe of another type or active galactic nuclei. When SNe Ia are observed within a week of maximum light, we find that we can spectroscopically identify most of them up to $z=1.1$. Beyond this redshift, very few candidates were spectroscopically identified as SNe Ia. The current generation of super red-sensitive, fringe-free CCDs will push this redshift limit higher.
\end{abstract}

Key words: stars: supernovae: general - cosmology: observations-surveys

\section{Introduction}

Type Ia supernovae (SNe Ia) have proven to be very good standard candles for cosmological studies. They are bright enough to detect at cosmological distances, $z \sim 1.5$, if one uses 8-10 m class ground-based optical telescopes or the Hubble Space Telescope (HST), and their luminosities can be standardised using empirical relations between luminosity and light curve shape (Phillips 1993) and colour (Tripp 1998).

$\mathrm{SNe}$ Ia have played a leading role in measuring the expansion history of the universe since two independent teams, the Supernova Cosmology Project (SCP) and the High- $z$ Team, discovered the accelerating expansion of the 
universe (Perlmutter et al. 1999; Riess et al. 1998). Since then, many projects to discover and identify SNe Ia have been organized. For example, the Carnegie Supernova Project (CSP; Hamuy et al. 2006), the Nearby Supernova Factory (SNfactory; Aldering et al. 2002), the HarvardSmithsonian Center for Astrophysics (CfA) supernova survey (Jha et al. 2006; Hicken et al. 2009), the Sloan Digital Sky Survey-II Supernova Survey (SDSS-II SN Survey; Sako et al. 2008; Frieman et al. 2008), the Supernova Legacy Survey (SNLS; Astier et al. 2006), the Equation of State: SupErNovae trace Cosmic Expansion (ESSENCE) survey (Miknaitis et al. 2007; Wood-Vasey et al. 2007), and Higher-Z team (Riess et al. 2007) have detected around $1000 \mathrm{SNe}$ up to redshift $z \sim 1.5$. The combination of SN Ia data with measurements of cosmic microwave background (CMB) fluctuations (Spergel et al. 2003; Spergel et al. 2007; Komatsu et al. 2009), baryon acoustic oscillations (BAO; Eisenstein et al. 2005) and galaxy cluster number counts (Vikhlinin et al. 2009) have constrained the dark energy equation of state parameter. However, the limits are consistent with very different dark energy models, so the fundamental nature of dark energy remains unclear. It is currently one of the biggest mysteries in physics, and combined astronomical observations (SNe Ia, CMB, BAO and weak lensing) seem to be the only way to constrain its properties.

Since the discovery of the accelerating expansion of the universe, the SCP has been carrying out imaging surveys for SNe Ia at $z \gtrsim 1$, an epoch during which the expansion of the universe is expected to be decelerating. Spectroscopic follow-up observations are an essential part of these surveys, providing spectroscopically determined redshifts and, when necessary and possible, direct confirmation of the SN type. In this paper, we present spectra of $\mathrm{SNe}$ and their host galaxies taken with the Faint Object Camera And Spectrograph (FOCAS; Kashikawa et al. 2002) on the Subaru 8.2-m telescope. Twelve SN candidates shown here were found in ground-based observations targeting blank fields and nearby galaxy clusters with Suprime-Cam (Miyazaki et al. 2002) on Subaru and the CFH12k camera (Cuillandre et al. 2000) on the Canada-France-Hawaii telescope (CFHT). The remaining 27 SN candidates were discovered using the Advanced Camera for Surveys (ACS; Benítez et al. 2003) on HST ${ }^{1}$ targeting high redshift galaxy clusters in a program called the HST Cluster SN Survey (Program number 10496, PI: Perlmutter). These SN searches specifically targeted $z>1$ $\mathrm{SNe} \mathrm{Ia}$, for which there have been relatively few spectroscopically confirmed SNe Ia (Aldering et al. 1998; Coil et al. 2000; Tonry et al. 2003; Barris et al. 2004; Riess et al. 2004; Lidman et al. 2005; Riess et al. 2007). In §2, we summarize the SN searches and present data for both imaging and spectroscopy. Spectroscopic data reductions

\footnotetext{
Based on observations made with the NASA/ESA Hubble Space Telescope and obtained from the data archive at the Space Telescope Institute. STScI is operated by the Association of Universities for Research in Astronomy, Inc. under the NASA contract NAS 5-26555. The observations are associated with program 10496.
}

are described in $\S 2$. SN and host galaxy classifications are shown in $\S 3$ and $\S 4$, respectively. In $\S 5$, we describe factors that influence the classification of SNe. $\S 6$ is a summary of the paper. We use the standard $\Lambda$ CDM cosmological parameters of $\left(H_{0}, \Omega_{M}, \Omega_{\Lambda}\right)=(70,0.27,0.73)$ for calculating the age of the universe at a certain redshift. All magnitudes are measured in the AB system.

\section{Observations and Data}

The spectroscopic observations described in this paper were carried out during our SN campaigns that were specifically targeting $z>1$ SNe Ia. Each campaign generally consisted of broad band imaging to discover SNe Ia, spectroscopic follow-up to determine SN types and redshifts, and photometric follow-up to measure light curves. To first find and then effectively follow $z>1$ SNe Ia, we used both wide-field imagers on ground-based telescopes, namely Suprime-Cam $\left(34^{\prime} \times 27^{\prime}\right)$ on the Subaru telescope and the CFH12k camera $\left(42^{\prime} \times 28^{\prime}\right)$ on CFHT, and ACS $\left(3.3^{\prime} \times 3.3^{\prime}\right)$ on HST. SN candidates shown in this paper were found during 2001, 2002, 2005 and 2006.

\subsection{SN Search Campaigns}

Our search for SNe Ia was conducted in a series of four campaigns. Three of these were conducted with ground based facilities during the Northern Spring of 2001, the Northern Spring of 2002 and the Northern Fall of 2002. The fourth was an ACS search conducted during 2005 and 2006. Some campaigns consisted of multiple searches with several instruments. Details of the searches are given in Table 1. Additional details can be found in Lidman et al. 2005, Yasuda et al. 2009, and Dawson et al. 2009, and are briefly summarised here. Some of the SN discoveries were reported in IAU circulars (Doi et al. 2001; Yasuda et al. 2002; Doi et al. 2003; Dawson et al. 2006).

\subsubsection{The Subaru 2001 Campaign}

The Subaru Suprime-Cam campaign conducted in the Spring of 2001 was a pilot search for later SN searches with this instrument. The relatively wide field-of-view and the high sensitivity provided by Suprime-Cam enables the discovery of high-redshift SNe more effectively than ACS (Yasuda et al. 2003). Only two- or threeepochs were taken with Suprime-Cam, which means that these data are not, by themselves, enough to derive light curves, although one candidate was followed with WFPC2 on HST (Amanullah et al. 2009). The search fields were centered on two galaxy clusters, CL 1604 $4321(z=0.90$; Lubin, Mulchaey, \& Postman 2004) and MS 1520.1+3002 $(z=0.117$; Stocke et al. 1991), and several blank fields, such as the Subaru Deep Field (SDF; Kashikawa et al. 2004). The SDF observations were carried out as a part of the SDF project. The imaging data for the SN searches were obtained in either the $R_{C}$ or $i^{\prime}$ filters. Typical exposure times and limiting magnitudes for each epoch were one hour and $R_{C}, i^{\prime} \sim 26$ mag. SNe found in these campaigns are named [field]-[SN ID] or [field][SN ID].

The Suprime-Cam data are very useful for investigating $\mathrm{SN}$ rates even though there are only 2 or 3 epochs. 
Table 1. Summary of the SN candidate search campaigns. The campaign name and search fields are denoted in columns 1 and 2. The telescope and instrument used for the SN search, and the number of pointings are denoted in columns 3 and 4 . The search season, the broad-band filter used in the search, the number of search epochs, and the number of spectra shown in this paper are denoted in column 5, 6, 7, and 8, respectively. $N_{\text {spec }}$ for HST Cluster Supernova Survey is the number of SN candidates and host galaxies.

\begin{tabular}{|c|c|c|c|c|c|c|c|}
\hline Campaign & Field & Telescope/Instrument & $N_{\text {pointings }}$ & Year/Month & Band & $N_{\text {epoch }}$ & $N_{\text {spec }}$ \\
\hline Subaru 2001 & CL1604_0,4 & Subaru/Suprime-Cam & 2 & $2001 / 04-2001 / 05$ & $R_{C}$ & 2 & 1 \\
\hline Subaru 2001 & MS1520.1 & Subaru/Suprime-Cam & 1 & $2001 / 04-2001 / 05$ & $i^{\prime}$ & 2 & 1 \\
\hline Subaru 2001 & SDF & Subaru/Suprime-Cam & 1 & $2001 / 04-2001 / 05$ & $i^{\prime}$ & 2 & 5 \\
\hline Spring 2002 & $\mathrm{SDF}$ & Subaru/Suprime-Cam & 2 & $2002 / 04-2002 / 05$ & $i^{\prime}$ & 2 & 0 \\
\hline Spring 2002 & $\mathrm{SDFe}, \mathrm{SDF} w$ & Subaru/Suprime-Cam & 2 & $2002 / 04-2002 / 05$ & $i^{\prime}$ & 2 & 0 \\
\hline Spring 2002 & $\mathrm{SS} 1,2,3,4$ & Subaru/Suprime-Cam & 4 & 2002/03-2002/04 & $i^{\prime}$ & 3 & 1 \\
\hline Spring 2002 & C02 fields & $\mathrm{CFHT} / \mathrm{CFH} 12 \mathrm{k}$ & 3 & 2002/03-2002/06 & $I$ & $\sim 10$ & 0 \\
\hline Fall 2002 & SXDF & Subaru/Suprime-Cam & 5 & 2002/09-2003/10 & $i^{\prime}$ & $5-7$ & 2 \\
\hline HST Cluster SN & galaxy clusters & $\mathrm{HST} / \mathrm{ACS}$ & 25 & $2005 / 08-2006 / 08$ & $z_{850}$ & $5-10$ & $8+5$ \\
\hline
\end{tabular}

By combining Suprime-Cam data in Abell 2152 (Totani et al. 2005), Oda et al. (2008) obtained SN rates for both $\mathrm{SNe}$ Ia and core-collapse $\mathrm{SNe}$, and, by using a theoretical model that relates SNe rate to the star formation rate (Oda \& Totani 2005), the cosmic star formation history. This provides an estimate that is independent of galaxy studies. It also takes advantage of the fact that SNe can trace star formation activity even though galaxies are either too diffuse or too faint to detect. Using well-sampled light curves from the SXDS data (Morokuma et al. 2008 and $\S .2 .1 .3$ of this paper), the cosmological evolution of the SN Ia rate can be measured (Totani et al. 2008; Ihara et al. 2009).

\subsubsection{The Spring 2002 Campaign}

The Spring 2002 Campaign consisted of four searches; two searches with Subaru, a CFHT search, and a CTIO search (Lidman et al. 2005). The two Subaru searches used Suprime-Cam, were done back-to-back and searched for SNe Ia in the SDF field, two fields surrounding the $\mathrm{SDF}$ field (SDFe and SDFw, which are to the east and west of the SDF, respectively), and four other blank fields (SS1, SS2, SS3, and SS4). The CFHT search was a rolling search, which was similar to the one subsequently done by the SNLS (Astier et al. 2006) where each field was observed 3 to 4 times per lunation over a period of several lunations. These kinds of searches automatically provide well-sampled light curves of discovered transients. We did not follow CTIO candidates with FOCAS. SNe discovered in this search are reported in Lidman et al. (2005).

SN candidates found in this campaign were named S02[SN ID] and C02-[SN ID] for the Subaru and CFHT searches, respectively. Followup imaging observations of several high-redshift SNe were obtained with HST/ACS and two ground based IR instruments: ISAAC (Infrared Spectrometer And Array Camera; Moorwood et al. 1998) on the VLT and NIRI (Near InfraRed Imager and Spectrometer; Hodapp et al. 2003) on Gemini-North. These observations will be reported in Suzuki et al. (in preparation)

\subsubsection{The Fall 2002 Campaign}

In the Fall 2002 campaign, we took multi-epoch $i^{\prime}$-band images of five fields with Suprime-Cam (Yasuda et al.
2009). The fields were centered on the Subaru/XMMNewton Deep Field (SXDF). The Subaru/XMM-Newton Deep Survey (SXDS) project (Sekiguchi et al. 2004; Sekiguchi et al. 2009) has taken deep multi-wavelength data, from X-ray to radio, of this region.

Compared to earlier Subaru campaigns, the search and follow-up of candidates discovered during the Fall 2002 campaign was more comprehensive. Reference images taken during the end of September 2002 were compared with search images that were taken during the beginning of November 2002. The typical exposure time was one hour and the limiting magnitude was $i^{\prime} \sim 26$ mag. The deep and wide-field Suprime-Cam data provided us with $\sim 100$ variable objects over a timescale of 1 month (Morokuma et al. 2008; Yasuda et al. 2009). Follow-up imaging observations of several high-redshift SNe were carried out using HST/ACS in the optical and NICMOS (Near Infrared Camera and Multi-Object Spectrometer; Thompson et al. 1999) on HST in the near infrared. We also obtained ground-based near-infrared $J$-band imaging data of one candidate with both CISCO (Cooled Infrared Spectrograph and Camera for $\mathrm{OH}$-airglow Suppressor (Iwamuro et al. 2001, Motohara et al. 2002)) on the Subaru telescope and VLT/ISAAC. These observations will be reported in Suzuki et al. (in preparation). SN candidates found in this campaign are named SuF02-[SN ID].

\subsubsection{The HST Cluster Supernova Survey}

The HST Cluster Supernova Survey was conducted in 2005 and 2006 with ACS on HST (Dawson et al. 2009). The targeted fields were galaxy clusters at $0.9<z<1.5$. Galaxy clusters are rich in early-type galaxies, which are expected to have little dust. In current SN Ia cosmological studies, the systematic uncertainty in the correction for dust attenuation is comparable to statistical uncertainties. For example, it is not clear if the extinction law is universal or unevolving. The most straightforward way to avoid (or reduce) this systematic error is to find SNe Ia in dust-free environments. Also, the dispersion in the corrected peak $B$-band luminosity of SNe Ia in early-type galaxies is smaller than that in late-type galaxies (e.g., Takanashi, Doi, \& Yasuda 2008). Therefore, we searched for SNe Ia in early-type galaxies by targeting galaxy clus- 
ters, which is an effective way of finding a large number of early type galaxies at high redshift in small fields of view. Another advantage in targeting early-type galaxies, where star-forming activities have been quenched, is that SNe found in such galaxies are expected to be SNe Ia with high probability, as there should be no core-collapse SNe in these galaxies.

In total, 25 high redshift galaxy clusters were targeted. Typical exposure times and limiting magnitudes for each epoch were 30 minutes and F850LP $\left(z_{850}\right) \sim 25$ mag, respectively. The clusters were also observed using the ACS F775W $\left(i_{775}\right)$ filter and active high redshift SNe were observed with the F110W filter on NICMOS. The SN candidates found in this campaign are named SCP[year][Cluster ID][SN ID] where "year" corresponds to the year of discovery, "Cluster ID" is a letter arbitrarily assigned to each cluster for scheduling purposes, and "SN ID" is a number assigned to each SN within a cluster according to its time of discovery in the search.

\subsection{FOCAS Follow-up Spectroscopic Observations}

Spectroscopic follow-up is used to obtain redshifts and, if the SN phase was near maximum light, the SN type. The candidates observed with FOCAS are summarized in Tables 2 and 3. Spectroscopic follow-up was also done with other facilities and is reported elsewhere (Lidman et al. 2005; Yasuda et al. 2009). During the Fall 2002 campaign, the Subaru telescope was used mainly for discovering SNe, so the number of SN candidates observed spectroscopically with FOCAS was small for this particular campaign.

We used the long slit mode of FOCAS until December 2005 and then mainly the multi-object slit mode after that. There are two reasons for the change. First, candidates from the HST Cluster Supernova Survey campaign were faint, which increased the difficulty in acquiring these targets with the long slit mode. The multi-object slitmasks can be manufactured to include several alignment holes to catch bright stars within the FOCAS FOV (6' diameter), thus enabling us to center targets more precisely. Slitmask observations also allow us to observe additional targets, such as cluster members, at the same time (Eisenhardt et al. 2008; Tanaka et al. 2008; Dawson et al. 2009; Meyers et al. 2009). Also, improvements in the mask alignment software by the FOCAS instrument team allowed us to acquire targets more quickly. Slit widths were $0 . \prime 8$ in most of the observations. When the seeing was poor, slit widths of $1^{\prime \prime} 0$ were used. The atmospheric dispersion corrector was used in all observations. Combinations of grisms and order-sort filters are 300B and SY47, or 300R and SO58, which result in spectra covering the $4700-9000 \AA$ and $5800-10000 \AA$ regions, respectively. Each exposure lasted 20 to 30 minutes. The objects were dithered by several arcseconds over several positions along the slit in order to effectively remove bad pixels, cosmic rays, and detector fringes. Exposure times varied with target brightness and priority. Typically, the

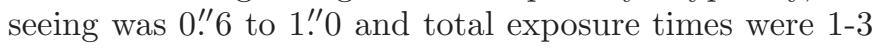
hours.

\subsection{Spectroscopic Data Reduction}

The FOCAS ${ }^{2}$ spectroscopic data were reduced in a mostly standard way using the Image Reduction and Analysis Facility $\left(\mathrm{IRAF}^{3}\right)$. The bias was removed by fitting a low order polynomial to the overscan region, which lies parallel to the dispersion direction, and the flatfielding was done with lamp flats. The background was removed while simultaneously masking bad pixels and bad columns, and correcting for detector fringing, except for those cases in which there were too few frames to compute a fringe correction. The flexure is small enough for one to use all the data taken with the same configuration during a night to make fringe correction images. The $2 \mathrm{~d}$ spectral data were then stacked and the $1 \mathrm{~d}$ spectrum of the object extracted. Wavelength calibration was done using night-sky emission lines. The flux scale was calibrated using the standard star spectrum obtained closest in time. BD+28d4211, Feige34, Feige110, Hz44, GD71, G191B2B, LTT2415, and Wolf1346 were used as flux standards. Most of them are HST spectrophotometric standard stars with well-measured flux densities. We also removed strong telluric absorption features using the standard star data that was used for flux calibration; however, we note that these corrections are not perfect, and when presenting the spectra we mark the main regions that may be affected by telluric features.

Fringes, which can be significant at long wavelengths, were removed from the sky background to increase the signal-to-noise ratio of the spectra. To remove these fringe patterns effectively, we adopted an observational and data reduction strategy that is described below. The procedure is similar to that described in Lidman et al. (2005), which was used for data obtained with FORS1 and FORS2 on the VLT. The difference between ours and Lidman et al. (2005) comes from the specific properties (distortion, pixel scale, spectral resolution, and so on) of each instrument.

All observations were dithered by several arcseconds along the spatial direction of the slits, which are set perpendicular to the dispersion direction. In long slit spectroscopy, we use all the exposures with the same configuration during a given night to create a fringe frame for that set up and that night. We also carefully designed slitmasks for SCP05D0 and SCP05D6 (D_mask1 and D_mask2), SCP05E12 (E_mask4 and E_mask6), and SCP05T1 (T_mask1 and T_mask2) so that the slit positions of these targets on the CCD were the same. Data were sorted with respect to grism, slit width, date and the location of the candidate on the $2 \mathrm{D}$ spectrum. Fringe frames were created by clipping deviant pixels (including pixels with flux from objects) and by averaging the reminder. Since the intensity of rows (the spatial axis of the spectra are along detector rows) can vary with respect to

2 For the Subaru 2001 Campaign a detector mosaic of two $2 \mathrm{k} \times 4 \mathrm{k}$ 3-side buttable CCDs fabricated by SITe were used. For all other campaigns, a mosaic of MIT/LL CCDs were used.

3 IRAF is distributed by the National Optical Astronomy Observatories, which are operated by the Association of Universities for Research in Astronomy, Inc., under cooperative agreement with the National Science Foundation. 
Table 2. Summary of FOCAS Observations. Column 1 denotes observing programs for FOCAS observations and allocated nights in parentheses. Column 2 denotes observing dates in yyyy $/ \mathrm{mm} / \mathrm{dd}$. Exposure times (in seconds), target names, spectroscopic modes, grism/order-sort filter configurations, and standard stars used for flux calibration are listed in columns 3, 4, 5, 6, and 7 . The letters "LS" indicate that the long-slit mode was used. When the multi-object spectroscopic mode was used, the slitmask name is used instead. The spectroscopic configuration was either 300B/SY47 or 300R/SO58, which provide spectra over the $4700-9000 \AA$ and $5800-10000 \AA$ wavelength ranges, respectively. In addition to the night listed below, 6 nights were lost to clouds.

\begin{tabular}{|c|c|c|c|c|c|c|}
\hline Program & Date (UT) & Exposure & Targets & Mode & Configuration & Standard Star \\
\hline S01A-079 & $2001 / 05 / 26$ & $600 \times 1$ & SDF1 & LS & $300 \mathrm{~B} / \mathrm{SY} 47$ & Feige34 \\
\hline \multirow{6}{*}{$(2)$} & & $2100 \times 3$ & SDF5 & LS & $300 \mathrm{~B} / \mathrm{SY} 47$ & Feige34 \\
\hline & $2001 / 05 / 27$ & $900 \times 1$ & CL1604_0-1 & LS & $300 \mathrm{~B} / \mathrm{SY} 47$ & Feige34 \\
\hline & & $2100 \times 2$ & $1520.1-2$ & LS & $300 \mathrm{~B} / \mathrm{SY} 47$ & Feige34 \\
\hline & & $2100 \times 3$ & SDF2 & LS & $300 \mathrm{~B} / \mathrm{SY} 47$ & Feige34 \\
\hline & & $2100 \times 1$ & SDF4 & LS & 300B/SY47 & Feige34 \\
\hline & & $900 \times 1$ & SDF6 & LS & $300 \mathrm{~B} / \mathrm{SY} 47$ & Feige34 \\
\hline \multirow{3}{*}{$\begin{array}{l}\text { S02A-174 } \\
\text { (1) }\end{array}$} & $2002 / 04 / 17$ & $1800 \times 3$ & S02-032 & LS & $300 \mathrm{~B} / \mathrm{SY} 47$ & Feige34 \\
\hline & & $1500 \times 1$ & C02-005 & LS & $300 \mathrm{~B} / \mathrm{SY} 47$ & Feige34 \\
\hline & & $1800 \times 1$ & $\mathrm{C} 02-007$ & LS & $300 \mathrm{~B} / \mathrm{SY} 47$ & Feige 34 \\
\hline \multirow{6}{*}{$\begin{array}{l}\text { S02B-I04 } \\
(2) \\
\text { S05B-137 } \\
(1.5)\end{array}$} & $2002 / 11 / 13$ & $2400 \times 3$ & SuF02-012 & $\mathrm{LS}$ & $300 \mathrm{R} / \mathrm{SO} 58$ & Feige110 \\
\hline & & $1800 \times 3$ & SuF02-061 & LS & $300 \mathrm{R} / \mathrm{SO} 58$ & Feige110 \\
\hline & $2005 / 09 / 26$ & $1800 \times 5$ & SCP05D0 & $\overline{L S}$ & $300 \mathrm{R} / \mathrm{SO} 58$ & LTT2415 \\
\hline & & $1800 \times 3$ & SCP05P1 & LS & $300 \mathrm{R} / \mathrm{SO} 58$ & LTT2415 \\
\hline & $2005 / 09 / 27$ & $1800 \times 2$ & SCP06N10 & LS & $300 \mathrm{R} / \mathrm{SO} 58$ & GD71 \\
\hline & $2005 / 09 / 28$ & $1800 \times 6$ & SCP05D6 & LS & $300 \mathrm{R} / \mathrm{SO} 58$ & GD71 \\
\hline \multirow{5}{*}{$\begin{array}{l}\text { S05B-137 } \\
(1.5)\end{array}$} & $2005 / 10 / 26$ & $1800 \times(4+2)^{*}$ & SCP05D6 & $\overline{L S}$ & $300 \mathrm{R} / \mathrm{SO} 58$ & $\mathrm{BD}+28 \mathrm{~d} 4211$ \\
\hline & & $1800 \times 3$ & SCP05P9 & LS & $300 \mathrm{R} / \mathrm{SO} 58$ & $\mathrm{BD}+28 \mathrm{~d} 4211$ \\
\hline & $2005 / 10 / 27$ & $1800 \times 4$ & SCP05D6 & LS & $300 \mathrm{R} / \mathrm{SO} 58$ & $\mathrm{BD}+28 \mathrm{~d} 4211$ \\
\hline & & $1800 \times 4$ & SCP05P9 & LS & $300 \mathrm{R} / \mathrm{SO} 58$ & $\mathrm{BD}+28 \mathrm{~d} 4211$ \\
\hline & $2005 / 10 / 28$ & $1800 \times 2$ & SCP06X13 & LS & $300 \mathrm{R} / \mathrm{SO} 58$ & GD71 \\
\hline S05B-137 (0.5) & $2005 / 12 / 27$ & $1800 \times 4$ & SCP06X18 & $\mathrm{LS}^{\dagger}$ & $300 \mathrm{R} / \mathrm{SO} 58$ & Feige34 \\
\hline S05B-137 & $2006 / 04 / 22$ & $1200 \times 5$ & SCP06F3, SCP06F6, SCP06F8 & F_mask2B & $300 \mathrm{R} / \mathrm{SO} 58$ & Feige34 \\
\hline \multirow{6}{*}{ (3) } & $2006 / 04 / 23$ & $1200 \times 5$ & SCP06X26, SCP06X27 & X_mask1 & $300 \mathrm{R} / \mathrm{SO} 58$ & Feige34 \\
\hline & & $1200 \times 5$ & SCP06H3 & H_mask2 & $300 \mathrm{R} / \mathrm{SO} 58$ & Feige34 \\
\hline & & $1200 \times 9$ & SCP06G3, SCP06G4 & G_mask1 & $300 \mathrm{R} / \mathrm{SO} 58$ & Wolf1346 \\
\hline & & $1200 \times 2$ & SCP06F3, SCP06F6, SCP06F8 & F_mask2B & $300 \mathrm{R} / \mathrm{SO} 58$ & $\mathrm{~Hz} 44$ \\
\hline & $2006 / 04 / 24$ & $1200 \times 2$ & SCP06X26, SCP06X27 & X_mask1 & $300 \mathrm{R} / \mathrm{SO} 58$ & Feige34 \\
\hline & & $1200 \times 10$ & SCP06G3, SCP06G4 & G_mask1 & $300 \mathrm{R} / \mathrm{SO} 58$ & Wolf1346 \\
\hline \multirow{4}{*}{$\begin{array}{l}\text { S05B-137 } \\
(1.5)\end{array}$} & $2006 / 06 / 28$ & $1200 \times 4$ & SCP06F6, SCP06F12 & F_mask2B & $300 \mathrm{~B} / \mathrm{SY} 47$ & Wolf1346 \\
\hline & & $1200 \times 2$ & SCP06L22 & LS & $300 \mathrm{~B} / \mathrm{SY} 47$ & $\mathrm{~Hz} 44$ \\
\hline & $2006 / 06 / 29$ & $1200 \times 8$ & SCP06A4 & A_mask1 & $300 \mathrm{R} / \mathrm{SO} 58$ & $\mathrm{BD}+28 \mathrm{~d} 4211$ \\
\hline & & $1200 \times 9$ & SCP06K0, SCP06K18 & K_mask1 & $300 \mathrm{R} / \mathrm{SO} 58$ & $\mathrm{~Hz} 44$ \\
\hline \multirow{4}{*}{$\begin{array}{l}\text { S06B-085 } \\
\text { (1) }\end{array}$} & $2006 / 08 / 23$ & $1200 \times 2$ & SCP06F6 & LS & $300 \mathrm{~B} / \mathrm{SY} 47$ & Wolf1346 \\
\hline & & $1200 \times 6$ & SCP06B3, SCP06B4 & B_mask1B & $300 \mathrm{R} / \mathrm{SO} 58$ & Wolf1346 \\
\hline & & $1200 \times 3$ & SCP06V6 & V_mask1 & $300 \mathrm{R} / \mathrm{SO} 58$ & G191B2B \\
\hline & & $1200 \times 6$ & SCP06N32, SCP06N33 & N_mask1 & $300 \mathrm{R} / \mathrm{SO} 58$ & G191B2B \\
\hline \multirow{4}{*}{$\begin{array}{l}\text { S06B-085 } \\
\text { (1) }\end{array}$} & $2006 / 12 / 24$ & $1200 \times 8$ & SCP05D0, SCP05D6 & D_mask1 & $300 \mathrm{R} / \mathrm{SO} 58$ & $\mathrm{BD}+28 \mathrm{~d} 4211$ \\
\hline & & $1200 \times 6$ & SCP05D0, SCP05D6 & D_mask2 & $300 \mathrm{R} / \mathrm{SO} 58$ & $\mathrm{BD}+28 \mathrm{~d} 4211$ \\
\hline & & $1200 \times 8$ & SCP06X18, SCP06X26, SCP06X27 & X_mask2 & $300 \mathrm{R} / \mathrm{SO} 58$ & Feige34 \\
\hline & & $1200 \times 4$ & SCP06E12 & E_mask3 & $300 \mathrm{R} / \mathrm{SO} 58$ & Feige34 \\
\hline \multirow{4}{*}{$\begin{array}{l}\text { S06B-085 } \\
\text { (2) }\end{array}$} & $2007 / 05 / 18$ & $1200 \times 6$ & SCP06E12 & E_mask4 & $300 \mathrm{R} / \mathrm{SO} 58$ & $\mathrm{~Hz} 44$ \\
\hline & & $1200 \times 6$ & SCP06T1 & T_mask1 & $300 \mathrm{R} / \mathrm{SO} 58$ & $\mathrm{BD}+28 \mathrm{~d} 4211$ \\
\hline & $2007 / 05 / 19$ & $1200 \times 7$ & SCP06E12 & E_mask6 & $300 \mathrm{R} / \mathrm{SO} 58$ & $\mathrm{~Hz} 44$ \\
\hline & & $1200 \times 7$ & SCP06T1 & T_mask2 & $300 \mathrm{R} / \mathrm{SO} 58$ & $\mathrm{BD}+28 \mathrm{~d} 4211$ \\
\hline
\end{tabular}

SCP05P9 was observed between the two exposures positions

$\dagger$ Slit positioning may have failed as there was no signal.

one another, each row is treated individually. The fringe frames were subtracted from the data after suitable scaling. Again, each row was treated individually. To help locate cosmic rays, an average sky spectrum was added back to the 2 dimensional fringe corrected data. These data were then registered and combined, after clipping for cosmic rays, and a spectrum of the candidate extracted.

\subsection{Spectral Fitting Method and SN Type Classification}

We determine SN types by fitting spectra with spectral templates using the software developed by Tokita (2009). The software fits the spectra over a grid of parameters: redshift, supernova template, extinction, and, if necessary, the host galaxy template and the fraction of host galaxy light. The templates consist of observed spectra of all SN types available from the literature, the FOCAS spectra of SDSS SNe (Zheng et al. 2008, Tokita 2009, Konishi et al.
2009), and synthetic spectra of SN Ia (Hsiao et al. 2007) and the other non-Ia types (Nugent et al. 2002). The software computes the reduced $\chi^{2}$ and determines the best combination of type, redshift, extinction, and epoch of each $\mathrm{SN}$ for which the reduced $\chi^{2}$ value is smallest. The extinction law used in this paper is from Cardelli, Clayton, and Mathis 1989. We also consider host galaxy contamination in some cases. Errors in the template spectrum are neglected.

When the redshift of the host, $z_{\mathrm{gal}}$, can be determined from host galaxy spectral features, we allow the redshift of the SN, $z_{\mathrm{SN}}$, to be fitted in the range $\Delta z \equiv\left|\left(z_{\mathrm{gal}}-z_{\mathrm{SN}}\right)\right| \sim$ $\pm 0.01-0.02$. The range allows for velocity variations found in normal $\mathrm{SNe}$ and, to a much smaller degree, a velocity offset due to the motion of the $\mathrm{SN}$ with respect to the host. When the light curve can be well fitted by light curve templates, differences between the epochs, $\Delta t$, 
Table 3. Summary of FOCAS spectrum fitting results. Columns 1 and 2 are target names. In column 2 , we list the IAU name, if the SN was reported in an IAU circular. Otherwise, this field is either left blank or is filled with the nicknames of the candidates. Columns 3 and 4 are the redshifts from the host galaxies and SNe. If we could classify a candidate as a SN, we report the name and epoch of the best-fitting local SN in columns 5 and 6, respectively. Epochs from the light curve fits are denoted in column 7 . The confidence index (C.I.) of the classification and the object type are listed in columns 8 and 9 , respectively. Notes on individual objects are in column 10 .

\begin{tabular}{|c|c|c|c|c|c|c|c|c|c|}
\hline Name 1 & Name 2 & $z_{\text {gal }}$ & $z_{\mathrm{SN}}$ & template & $t_{\mathrm{sp}}$ & $t_{\mathrm{LC}}{ }^{*}$ & C.I. $^{\dagger}$ & Type ${ }^{\dagger}$ & Notes \\
\hline CL1604_0-1 & SN 2001cq & - & 0.36 & SN 1998aq & -8 & - & 5 & Ia & Si II,S II. \\
\hline $1520.1-2$ & SN 2001cw & 0.94 & 0.95 & Hsiao Ia & -6 & -5 & 3 & $\mathrm{Ia}^{*}$ & $4000 \AA$ break. \\
\hline SDF1 & SN 2001cs & 0.431 & 0.42 & SN 1994D & -1 & - & 5 & Ia & Ca II $\mathrm{H}$ and $\mathrm{K}$. Sig. host galaxy contamination. \\
\hline SDF2 & SN 2001ct & - & ? & - & - & - & 1 & II? & Blue continuum. \\
\hline SDF4 & SN 2001cr & - & $?$ & - & - & - & 2 & $?$ & - \\
\hline SDF5 & SN 2001cv & 1.039 & 1.02 & Nugent Ia & 20 & - & 2 & $?$ & {$[\mathrm{O} \mathrm{II}]^{\S}$} \\
\hline SDF6 & SN 2001cu & 0.515 & $\begin{array}{l}0.50 \\
0.50\end{array}$ & $\begin{array}{l}\text { Nugent Ib/c } \\
\text { Hsiao Ia }\end{array}$ & $\begin{array}{l}-6 \\
-6\end{array}$ & - & 2 & $?$ & $\begin{array}{l}\text { Ca II H and K. Sig. host galaxy contamination. } \\
\text { Sig. host galaxy contamination. }\end{array}$ \\
\hline S02-032 & SN $2002 \mathrm{ff}$ & - & 1.02 & Nugent Ia & 0 & 0 & 4 & Ia & - \\
\hline C02-005 & - & 0.426 & - & - & - & - & 0 & $\mathrm{SB} / \mathrm{AGN}$ & Starburst or AGN. \\
\hline $\mathrm{C} 02-007$ & - & 1.780 & - & - & - & - & 0 & AGN & AGN. \\
\hline SuF02-012 & SN 2002lc & - & $1.22 ?$ & - & - & -1 & 2 & $?$ & Sig. host galaxy contamination. \\
\hline SuF02-061 & - & 1.085 & & - & - & $\sim 5$ & 2 & $?$ & Sig. host galaxy contamination. AGN? \\
\hline SCP06A4 & Aki & 1.19 & - & - & - & 0 & 2 & $?$ & Low signal-to-noise ratio. \\
\hline SCP06B3 & Isabella & 0.744 & - & - & - & 13 & 2 & $?$ & {$[\mathrm{O} \mathrm{II}], \mathrm{H} \beta,[\mathrm{O} \mathrm{III}]$} \\
\hline SCP06B4 & Michaela & 1.117 & - & - & - & 4 & 2 & $?$ & [O II], [Ne III]. \\
\hline \multirow[t]{2}{*}{ SCP05D0 } & Frida & 1.015 & - & - & - & 16 & - & - & $\mathrm{Ca}$ II $\mathrm{H}$ and $\mathrm{K}$. \\
\hline & & & - & - & - & 241 & - & - & Ca II H and K. Table 4. \\
\hline \multirow[t]{3}{*}{ SCP05D6 } & Maggie & 1.315 & - & - & - & -8 & 2 & $?$ & $\mathrm{Ca}$ II $\mathrm{H}$ and $\mathrm{K}$. \\
\hline & & & - & - & - & 4 & 2 & $?$ & $\mathrm{Ca}$ II $\mathrm{H}$ and $\mathrm{K}$. \\
\hline & & & - & - & - & 187 & - & - & Ca II H and K. Table 4. \\
\hline \multirow[t]{2}{*}{ SCP06E12 } & - & - & - & - & - & $>300$ & - & - & Nearby galaxy at $\mathrm{z}=1.025$. \\
\hline & & & - & - & - & $>480$ & - & - & - \\
\hline SCP06F3 & - & $1.21 ?$ & - & - & - & 0 & 2 & $?$ & An emission line at $6195 \AA ?$ AGN? \\
\hline \multirow[t]{3}{*}{ SCP06F6 } & - & - & - & - & - & -25 & 0 & non-Ia & Blue continuum. Barbary et al. 2009. \\
\hline & & & - & - & - & 42 & 0 & - & Weak continuum. \\
\hline & & & - & - & - & 98 & 0 & - & Very low signal-to-noise ratio. \\
\hline SCP06F 8 & Ayako & $0.789 ?$ & - & - & - & 40 & - & - & Spectrum dominated by the neighbor. \\
\hline SCP06F12 & Caleb & - & - & - & - & 194 & - & - & Almost no continuum. \\
\hline SCP06G3 & Brian & 0.962 & - & - & - & 24 & - & - & {$[\mathrm{O} \mathrm{II}]^{\S}$} \\
\hline SCP06G4 & Shaya & 1.350 & 1.35 & Hsiao Ia & -1 & -5 & 3 & $\mathrm{Ia}^{*}$ & Probable Ca II H and K. Table 4. \\
\hline $\mathrm{SCP} 06 \mathrm{H} 3$ & Elizabeth & 0.851 & 0.84 & Hsiao Ia & 2 & 0 & 4 & Ia & {$[\mathrm{O}$ II $],[\mathrm{O}$ III $]$} \\
\hline SCP06K0 & Tomo & 1.416 & - & - & - & 68 & - & - & $4000 \AA$ break. Table 4 \\
\hline SCP06K18 & - & 1.411 & - & - & - & 84 & - & - & $4000 \AA \AA$ break. Table 4 . \\
\hline SCP06L22 & - & 1.369 & - & - & - & - & 0 & AGN & broad Mg II. \\
\hline $\mathrm{SCP} 06 \mathrm{~N} 10^{\ddagger}$ & - & 0.203 & - & - & - & $>54$ & - & - & {$[\mathrm{O}$ III $], \mathrm{H} \alpha$} \\
\hline SCP06N32 & - & - & - & - & - & $<3$ & 2 & $\mathrm{Ib} / \mathrm{c} ?$ & - \\
\hline SCP06N33 & Naima & 1.189 & - & - & - & 4 & 2 & $?$ & Very low signal-to-noise ratio. \\
\hline SCP05P1 & Gabe & 0.926 & - & - & - & 16 & - & - & {$[\mathrm{O}$ II $],[\mathrm{O}$ III $]$} \\
\hline SCP05P9 & Lauren & 0.821 & 0.81 & Hsiao Ia & -2 & -3 & 3 & $\mathrm{Ia}^{*}$ & Sig. host galaxy contamination. [O II], [O III],H $\beta$. \\
\hline SCP06T1 & Jane & $1.112 ?$ & - & - & - & 450 & - & - & Probable $[\mathrm{O}$ II] \\
\hline SCP06V6 & - & 0.903 & - & - & - & - & 0 & AGN & [O II],Fe II,broad $\mathrm{H} \beta,[\mathrm{O}$ III]. \\
\hline SCP06X13 & - & 1.642 & - & - & - & - & 0 & AGN & Broad Mg II. \\
\hline \multirow[t]{2}{*}{ SCP06X18 } & - & - & - & - & - & 16 & 2 & $?$ & Almost no continuum. \\
\hline & & & - & - & - & 378 & - & - & Almost no continuum. \\
\hline \multirow[t]{2}{*}{ SCP06X26 } & Joe & $1.44 ?$ & - & - & - & 67 & - & - & A marginal emission line at $9100 \AA \AA$. \\
\hline & & & - & - & - & 312 & - & - & - \\
\hline \multirow[t]{2}{*}{ SCP06X27 } & Olivia & 0.435 & - & - & - & 38 & - & - & $\mathrm{Na}$ I D, H $\alpha,[\mathrm{N}$ II $]$. \\
\hline & & & - & - & - & 208 & - & - & $\mathrm{Na}$ I D, $\mathrm{H} \alpha,[\mathrm{N}$ II $]$ \\
\hline
\end{tabular}

which are in observer frame days.

$\dagger$ Generally left as "-" for targets that were observed more than two rest frame weeks after maximum light, where the host galaxy was the primary target.

$\ddagger$ No fringe corrections were applied. A 1!'0-width slit was used.

$\S$ The single emission line is identified as [O II]. 
are expected to be within $\sim \pm 3$ days. We note that epochs of spectra $t$ and $\tau$ are defined as days from maximum light and explosion, respectively, and that $t$ is generally applied for SNe Ia while $\tau$ is applied for core-collapse SNe. Larger differences of epochs are possible for later phases because the number of SN spectral templates that are available is not so large. For most of SN candidates discovered during the Subaru 2001 and Spring 2002 campaigns, there are too few light curve epochs to set strong constraints on the spectroscopic epoch.

In some cases, the extracted SN spectrum is contaminated by host galaxy light. If the contamination is significant, we remove the host galaxy light using one of two methods. In the case that the SN and the host galaxy are well resolved, we subtract a spectrum that is extracted from a region that is located on the other side of the center of the host. We assume axial symmetry of the host galaxy. When the SN is located close to the center of the host galaxy, we subtract a synthetic spectrum computed from the spectral library of Bruzual \& Charlot 2003 (hereafter BC03). In the second case, the amount of host contamination is estimated by minimizing the reduced $\chi^{2}$. When the SN is almost free from contamination of the host galaxy, judged from a visual inspection of the imaging and spectroscopic data, we do not make any corrections for the host galaxy.

We quantify the degree of confidence that we have correctly classified a candidate as a SN Ia using the index described in Howell et al. (2005). A confidence index (C.I.), ranging from 5 to 0 , is assigned to each $\mathrm{SN}$ candidate that was observed within two rest frame weeks of maximum light. In addition to the spectra, information from the lightcurve and the host, if available, are used to set this index. Candidates with 5, 4, 3, 2, 1, and 0 are certain SN Ia, highly probable SN Ia, probable SN Ia, unknown object, probably not a SN Ia, and not a SN Ia, respectively. For those candidates that are classified as SNe Ia with C.I. of 5, 4, and 3, we list the best matching template in Table 3 and plot the candidate and the best matching template in the figures of the following section. We also make this comparison for selected candidates with C.I. of 2. Following Howell et al. (2005), SNe Ia that are assigned a C.I. of 4 or 5 are typed as SN Ia, and those that are assigned a C.I. of 3 are typed as $\mathrm{SN} \mathrm{Ia}^{*}$, which indicates a higher degree of uncertainty for these SNe. No classification is made for candidates that were observed more than two weeks after maximum light, where the host galaxy was the primary target.

As a cross check of the classification, all spectra were fitted with superfit (Howell et al. 2005) and independently classified by two of the authors. In nearly all cases, the classifications match. For those few cases in which there was disagreement, always small, we usually adopted the more conservative classification.

The fitting results are summarized in Table 3.

\section{SN Type Classification Results}

In the following sections, we show spectra of 8 candidates from the Subaru 2001 and Spring 2002 search campaigns, 2 spectra from the Fall 2002 search campaign in the SXDS fields, and 10 spectra from the HST Cluster Supernova Survey. The confidence in the spectral classifications varies from 5 , a secure $\mathrm{SNe}$ Ia (SDF1, for example), to 0, not a SN Ia (SCP06F6, for example). The spectra of candidates that are clear AGN (C02-007 and SCP06V6, for example) are not shown. Also, the FOCAS spectrum of the extraordinary transient SCP06F6 was presented in Barbary et al. 2009 and is not re-shown here. All the candidates observed with FOCAS are summarised in Tables 2 and 3.

For display purposes only, the spectra shown in the following figures were smoothed with a Gaussian (FWHM of $\sim 30$ pixels or $\sim 40 \AA$ ) with each point weighted with the inverse of its variance. Although telluric absorption features were removed using the spectra of bright stars, the correction is not perfect, so these regions are highlighted in gray. Also highlighted are residuals from the strong atmospheric emission lines, such as those from Oxygen (5577 $\AA$ and $6300 \AA)$ and Sodium $(5890 \AA)$. Non-stellar features, such as the $[\mathrm{O} \mathrm{II}]$ emission line, which are not reproduced by BC03 models, are also marked. These regions can bias spectral fitting results, so they are masked out. The Suprime-Cam and ACS finding charts are $8^{\prime \prime} \times 8^{\prime \prime}$ and $5^{\prime \prime} \times 5^{\prime \prime}$, respectively. North is up and East is to the left.

\subsection{Candidates from the Subaru Spring Searches}

All the SN candidates shown in this section were found with Subaru Suprime-Cam in the springs of 2001 and 2002.

\subsubsection{CL1604_0-1}

CL1604_0-1 (SN 2001cq) was found in the field surrounding the galaxy cluster CL1604. On May 27, 2001, we obtained the spectrum with an exposure of $900 \mathrm{~s}$. The host galaxy can not be detected in the images. The spectrum (Figure 1) does not show any significant spectral features from the host galaxy and $z_{\text {gal }}$ can not be determined. Clear Si II $4000 \AA$, S II "w", and Si II $6150 \AA$ features are detected, indicating that this is a secure SN Ia at $z_{\mathrm{SN}}=0.36$. The C.I. is 5 . The best-fit SN spectrum is a normal SN Ia, SN 1998aq, at $t=-8$ days.

\subsubsection{1-2}

1520.1-2 (SN 2001cw) was found in the field centered on the galaxy cluster 1520.1. The FOCAS spectrum (Figure 2) was obtained on May 27, 2001 and the exposure was 4200 seconds. The SN is well separated from the probable host, which is located to the northeast of the $\mathrm{SN}$. The redshift of this galaxy is $z_{\text {gal }} \sim 0.94$ as determined from the $4000 \AA$ break. The spectrum can fitted with both the Nugent Type Ib/c template at $t=-4$ days redshifted to $z_{\mathrm{SN}}=0.95$ and the Hsiao Type Ia template at $t=-6$ days redshifted to $z_{\mathrm{SN}}=0.95$. The light curve is consistent with that of a SN Ia (Amanullah et al. 2009) and also $t_{\mathrm{sp}}$ matches $t_{\mathrm{LC}}$. We conclude that it is a probable SN Ia and set the C.I. to 3 . 

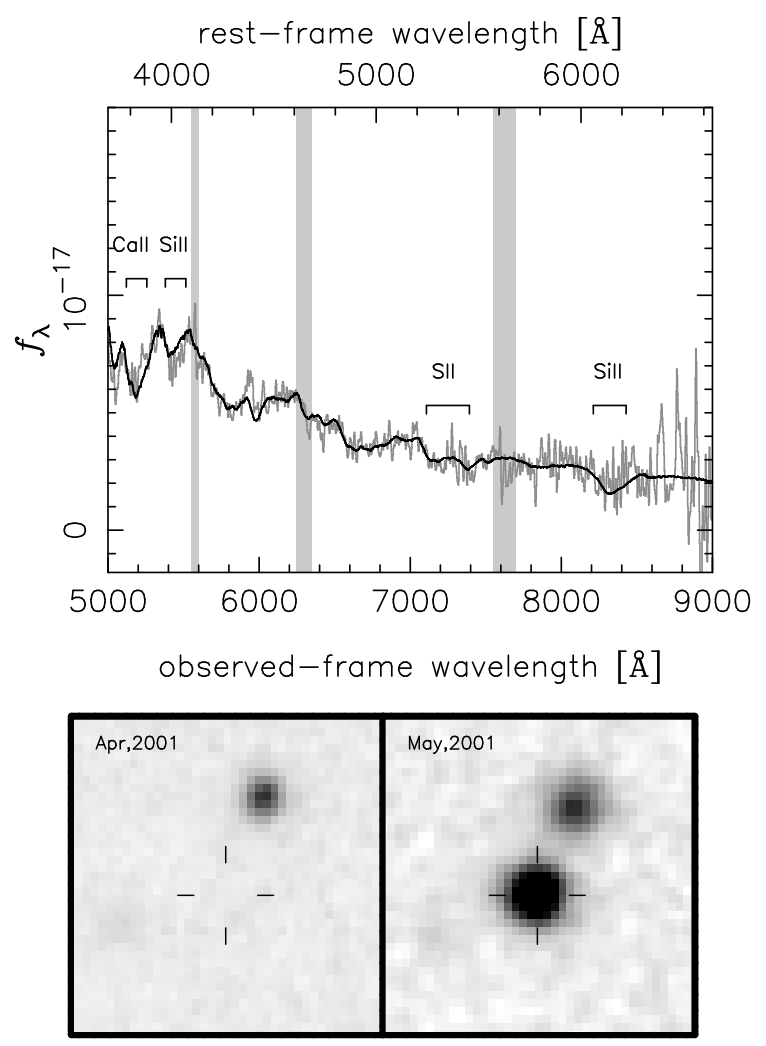

Fig. 1. (Top): The spectrum of CL1604_0-1 (SN 2001cq) at $z_{\mathrm{SN}}=0.36$ (gray) and the redshifted spectrum of SN 1998aq at $t=-8$ day (black). The host galaxy redshift $z_{\text {gal }}$ can not be determined. Light gray regions are masked because of atmospheric absorption or missubtraction of the sky background. The C.I. is 5. (Bottom): Finding charts of CL1604_0-1. The size is $8^{\prime \prime} \times 8^{\prime \prime}$. North is up and east is left.

\subsection{3. $S D F 1$}

SDF1 (SN 2001cs) was found during the 2001 run of the SDF project together with other SDF SN candidates (SDF2, SDF4, SDF5, and SDF6). This SN is only slightly offset from the center of the relatively bright host. Not surprisingly, the SN spectrum is strongly contaminated by host galaxy light. The 600 second spectrum (Figure 3) taken on May 26, 2001 indicates that the host galaxy is at $z_{\text {gal }}=0.431$ from $\mathrm{Ca}$ II $\mathrm{H}$ and $\mathrm{K}$ absorption lines. After subtracting a model of the host galaxy spectrum and correcting for extinction $(\mathrm{E}(B-V)=0.2 \mathrm{mag})$, the distinctive Si II feature of SNe Ia around $4000 \AA$ in the rest frame can be seen. This is a SN Ia with the C.I. of 5 at $z_{\mathrm{SN}}=0.42$. The best-fit spectrum is SN $1994 \mathrm{D}$ at $t=-1$ days.

\subsubsection{SDF2}

The spectrum of SDF2 (SN 2001ct) is shown in Figure 4. It was taken on May 27, 2001 and has a total integration time of 6300 seconds. This SN appears on a diffuse galaxy. The redshift of the likely host could not be determined and the spectrum of the SN is featureless and blue. We can determine neither the type nor the redshift of SDF2; however, the blue continuum suggests that this candidate is probably a Type II SNe. The C.I. is 1.
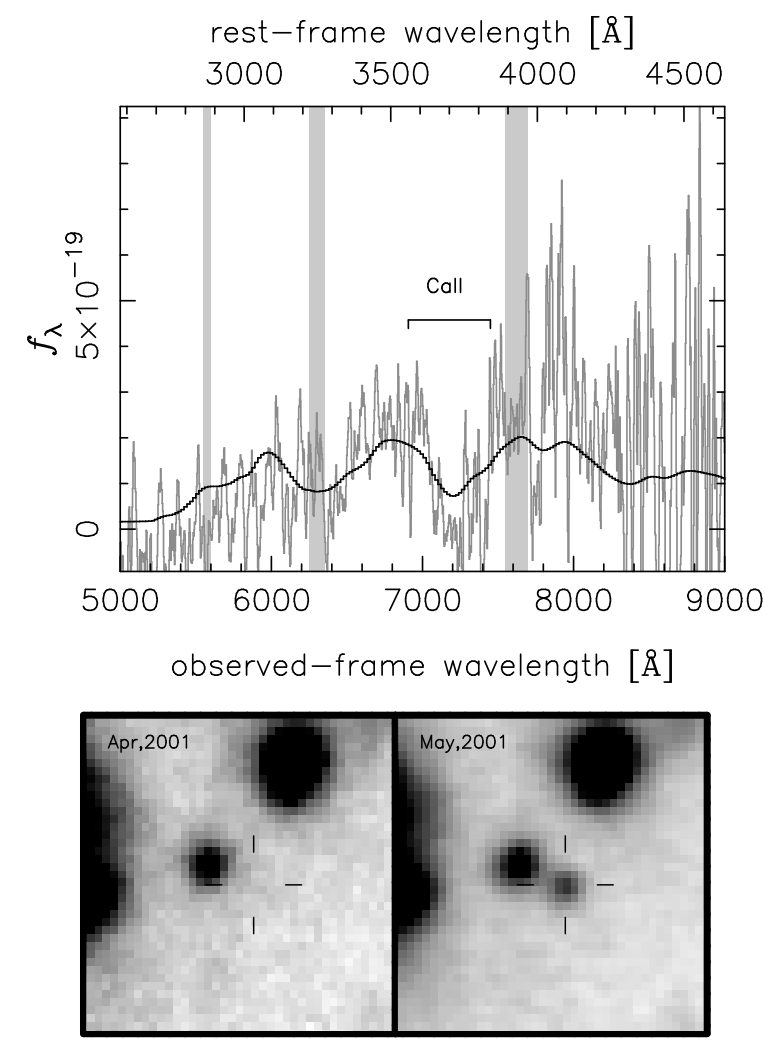

Fig. 2. (Top): The spectrum of 1520.1-2 at $z_{\text {gal }} \sim 0.94$ (gray) and the Hsiao Ia template at $t=-6$ days (black) redshifted to $z_{\mathrm{SN}}=0.95$. The C.I. is 3. (Bottom): Finding charts of 1520.1-2.

\subsection{5. $S D F 4$}

The spectrum of the unusual transient SDF4 (SN 2001cr) is shown in Figure 5. It was taken on May 27, 2001 and has a total integration of 2100 seconds. The Suprime-Cam reference image taken in April 2002 does not show any significant signal from the either the SN or the host. The SN component dominates the spectrum. The redshift of the host galaxy $z_{\text {gal }}$ could not be determined. There are no SN templates that fit the observed spectrum well. The best-fit SN template is the Nugent $\mathrm{SN} \mathrm{Ib/c} \mathrm{hypernova} \mathrm{(HN)} \mathrm{template} \mathrm{at} t=0$ days redshifted $z_{\mathrm{SN}}=0.78$. But some bumpy structures in the spectrum do not match well. A SN $1991 \mathrm{~T}$ template at $t=-11$ days redshifted to $z_{\mathrm{SN}}=0.81$ might fit the spectrum better although the $\chi^{2}$ values are slightly larger. The C.I. is 2.

\subsubsection{SDF5}

The spectrum of SDF5 (SN 2001cv) is shown in Figure 6. It was taken on May 26, 2001 and has a total integration of 5300 seconds. The $\mathrm{SN}$ is separated from the host galaxy and the SN component dominates the spectrum at the location of the SN. The redshift of the host is $z_{\text {gal }}=1.039$ as measured from a single strong emission line, which we identify as [O II]. It is very unlikley that the line is $\operatorname{Ly} \alpha$, as the line is not asymmetric and the continuum across the line shows no evidence for a discontinuity. It is unlikely to be $\mathrm{H} \alpha$ or [O III] as no other lines are seen, despite the strength of the detected line. When the spectral 

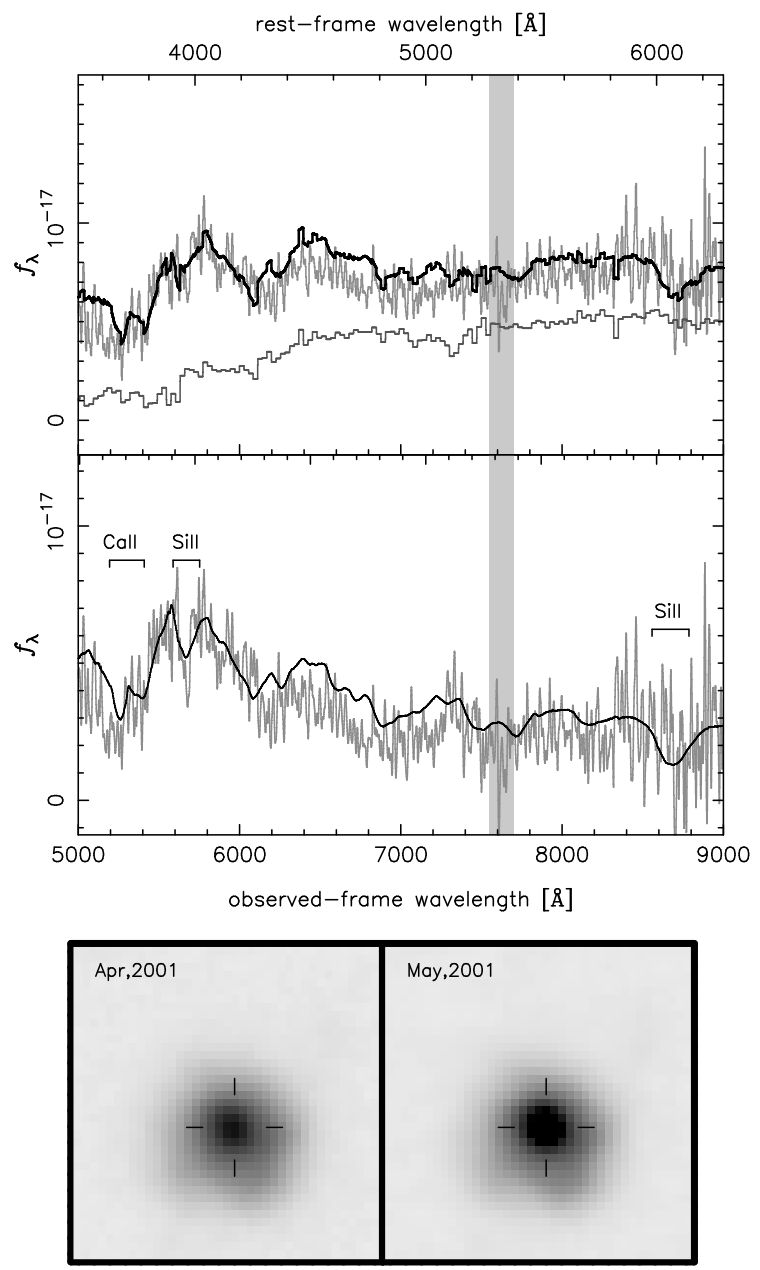

Fig. 3. (Top): In the upper panel, the spectrum of SDF1 (SN 2001cs) at $z_{\text {gal }}=0.431$ (gray), the galaxy template subtracted spectrum (dark gray), and the combined SN template plus galaxy template spectrum (black). In the lower panel, the galaxy-subtracted spectrum (gray) and and the spectrum of the best matching local SN, SN 1994D at $t=-1$ days, redshifted to $z_{\mathrm{SN}}=0.42$ (black). The C.I. is 5. (Bottom): Finding charts of SDF1.

template of a star forming galaxy is subtracted, the SN spectral fitting indicates that the Hsiao template SN Ia at $t=9$ days at $z_{\mathrm{SN}}=1.02$ is the best match. However, the spectral features characteristic of a SN Ia are marginal and we do not have a well-sampled light curve. Therefore, we set the C.I. to 2.

\subsubsection{SDF6}

The spectrum of SDF6 (SN 2001cu) is shown in Figure 7. It was taken on May 27, 2001 and has a total integration time of 900 seconds. The host is similar in brightness to the $\mathrm{SN}$, so the $\mathrm{SN}$ spectrum is contaminated by light from the host. A redshift of $z_{\text {gal }}=0.515$ is measured from the CaII $\mathrm{H}$ and $\mathrm{K}$ absorption lines of the host galaxy. After subtracting a passively evolving model for the spectrum of the host galaxy and correcting for extinction $(E(B-V)=0.2 \mathrm{mag})$ both the Nugent $\mathrm{Ib} / \mathrm{c}$ template at $t=-6$ days redshifted to $z_{\mathrm{SN}}=0.50$ and the
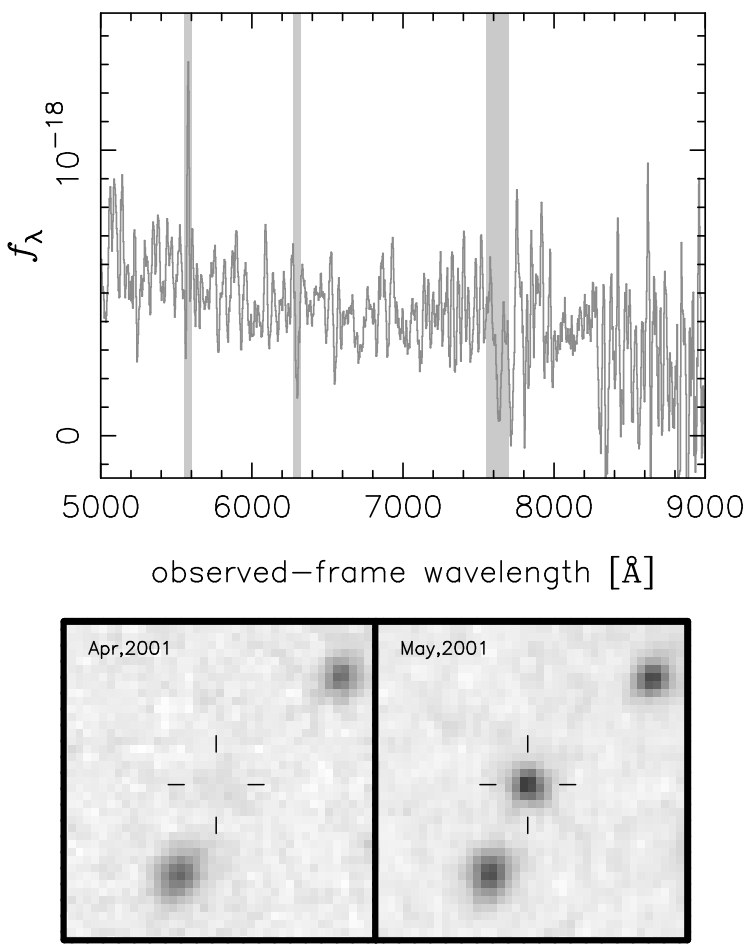

Fig. 4. (Top): Spectrum of SDF2 (SN 2001ct). The C.I. is 1. (Bottom): Finding charts of SDF2.
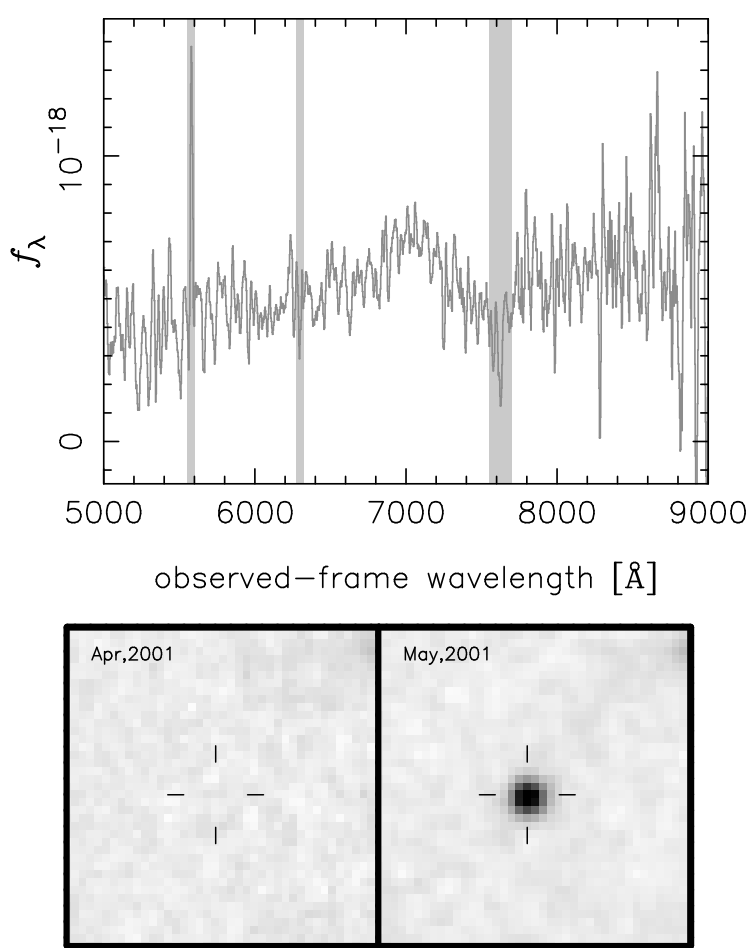

Fig. 5. (Top): Spectrum of SDF4 (SN 2001cr). The C.I. is 2. (Bottom): Finding charts of SDF4. 

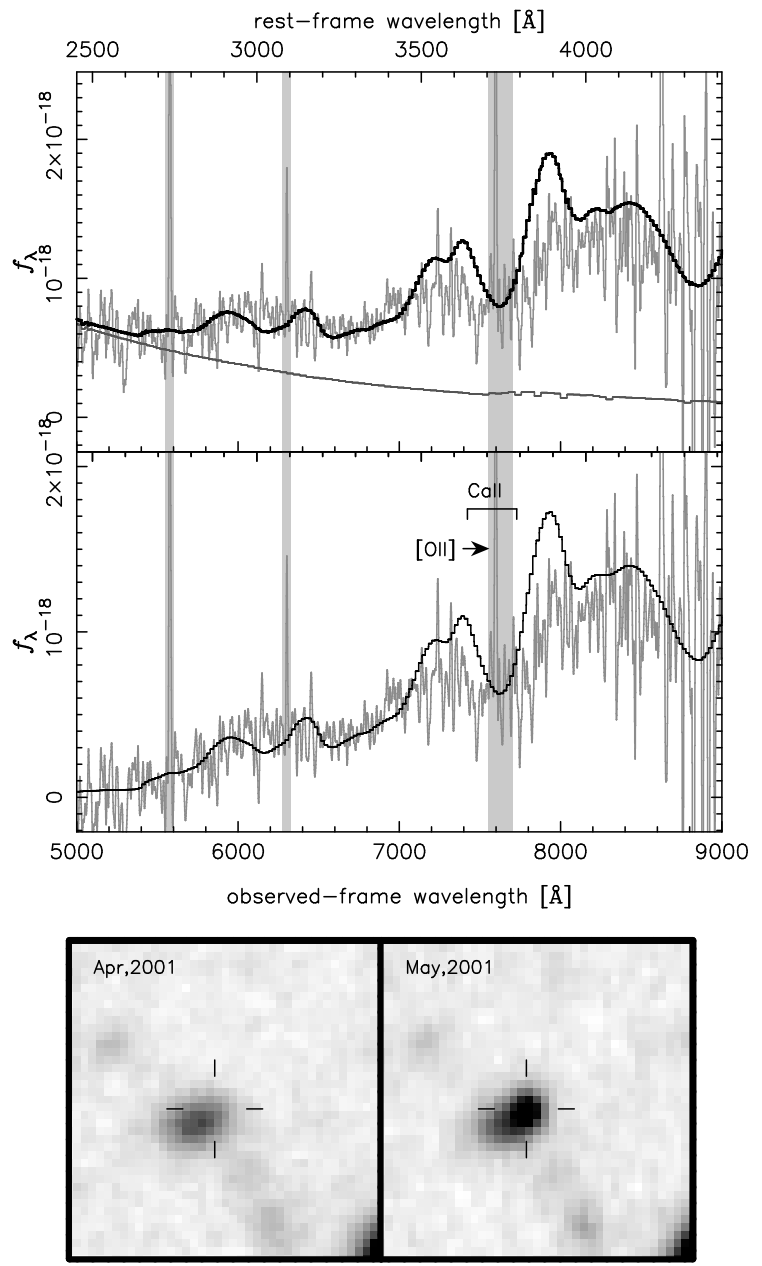

Fig. 6. (Top): The spectrum of SDF5 (SN 2001cv) at $z_{\text {gal }}=1.039$ (gray) and the Hsiao SN Ia template at $t=9$ days redshifted to $z_{\mathrm{SN}}=1.02$ (black). The C.I. is 2. (Bottom): Finding charts of SDF5.

Hsiao Ia template at $t=-6$ days redshifted to $z_{\mathrm{SN}}=0.50$ are acceptable fits to the spectrum. Since there a too few light curve points to help with the classification, the C.I. is set to 2 .

\subsubsection{S02-032}

The spectrum of S02-032 (SN 2002ff) is shown in Figure 8. It was taken on April 17, 2002 and has a total integration time of 5400 seconds. This SN appears on a diffuse galaxy whose redshift could not be measured. The SN component dominates the spectrum. The SN spectral fitting indicates that this is a $\mathrm{SN}$ Ia at $z_{\mathrm{SN}}=1.02$. The best-fit spectrum template is the Nugent normal Ia template at $t=0$ days. The light curve is consistent with that of a SN Ia and $t_{\mathrm{sp}}$ matches $t_{\mathrm{LC}}$. The C.I. is 4 .

\subsection{Candidates in the SXDS field}

All the SN candidates shown in this section were found with Subaru Suprime-Cam during the SN campaign conducted during the Fall of 2002.
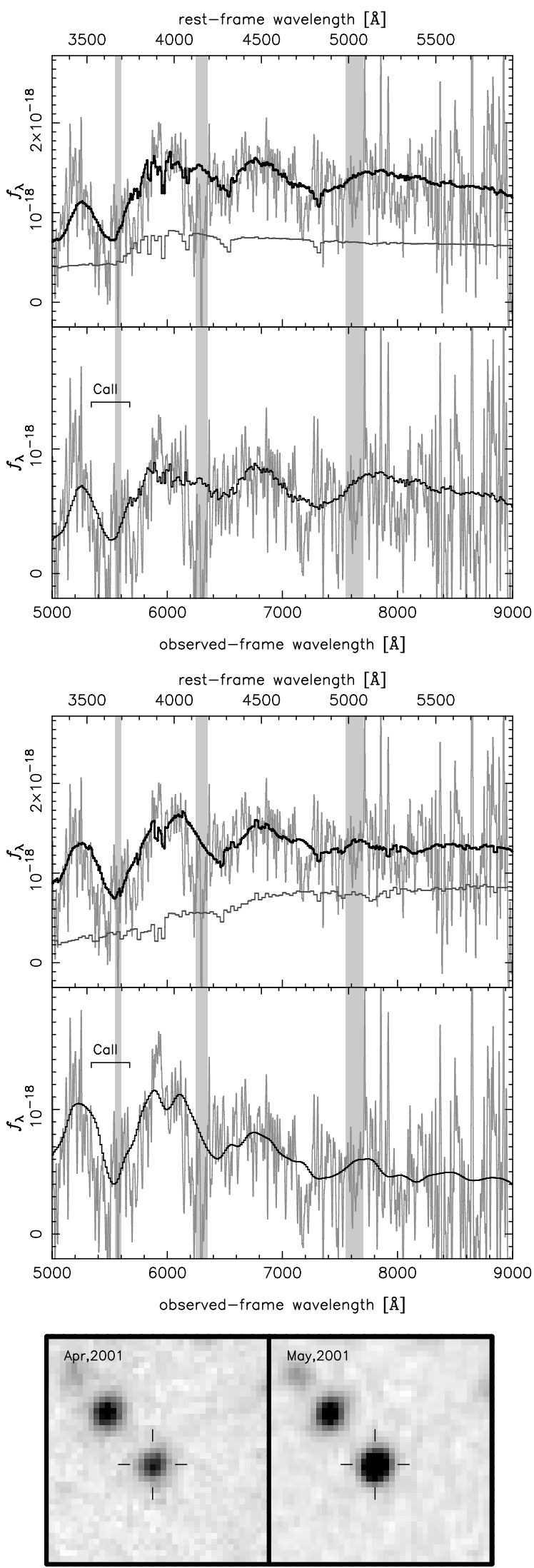

Fig. 7. (Top): The spectrum of SDF6 (SN 2001cu) at $z_{\text {gal }}=0.515$ (gray) and the Nugent $\mathrm{Ib} / \mathrm{c}$ template at $t=-6$ days redshifted to $z_{\mathrm{SN}}=0.50$ (black). (Middle): Spectrum of SDF6 at $z_{\text {gal }}=0.515$ (gray) and the Hsiao Ia template at $t=-6$ days redshifted to $z_{\mathrm{SN}}=0.50$ (black). The C.I. is 2. (Bottom): Finding charts of SDF6. 

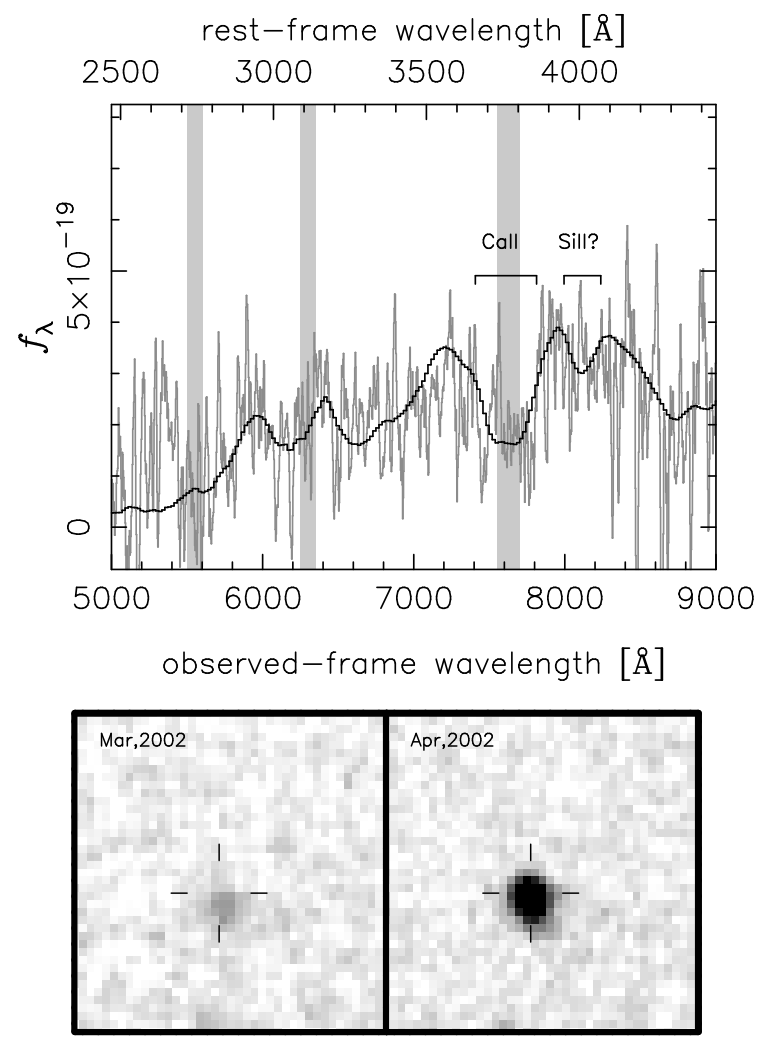

Fig. 8. (Top): The spectrum of S02-032 (SN 2002ff) at $z_{\mathrm{SN}}=1.02$ (gray) and the Nugent Ia template at $t=0$ days (black) redshifted to $z_{\mathrm{SN}}=1.02$. The C.I. is 4. (Bottom): Finding charts of S02-032.

\subsubsection{SuF02-012}

The spectrum of SuF02-012 (SN 2002lc) is shown in Figure 9. It was observed on November 13, 2002 and has a total integration time of 7200 seconds. It is hosted by a diffuse galaxy, whose redshift could not be determined. The spectral fitting indicates that it might be a SN Ia at $z_{\mathrm{SN}}=1.22$. However, the phase of the best-fit template, SN 1991T at $t=12$ days, disagrees with the light curve phase. SNe Ia at earlier phases and higher redshifts are also a possibility. Given the uncertainty, we have set the C.I. to 2. This target was a high priority object and spectroscopically observed with FORS2 on the ESO VLT, ESI on the Keck, and the ACS grism on the HST. All the spectra suggest that this is a high redshift SN. A future analysis of the combined data set may lead to this candidate being reclassified.

\subsubsection{SuF02-061}

The spectrum of SuF02-061 is shown in Figure 10. It was taken on November 13, 2002 with a total exposure time of 5400 seconds. This SN appears on a diffuse galaxy that has a redshift of $z_{\mathrm{gal}}=1.085$, as measured from several emission lines, including [O II], [NeIII], and some Balmer lines. The spectrum is too noisy or the host light dominates too much to classify the SN type. This galaxy is also detected in X-rays, suggesting that the variability may originate from AGN activity. The C.I. is 2.
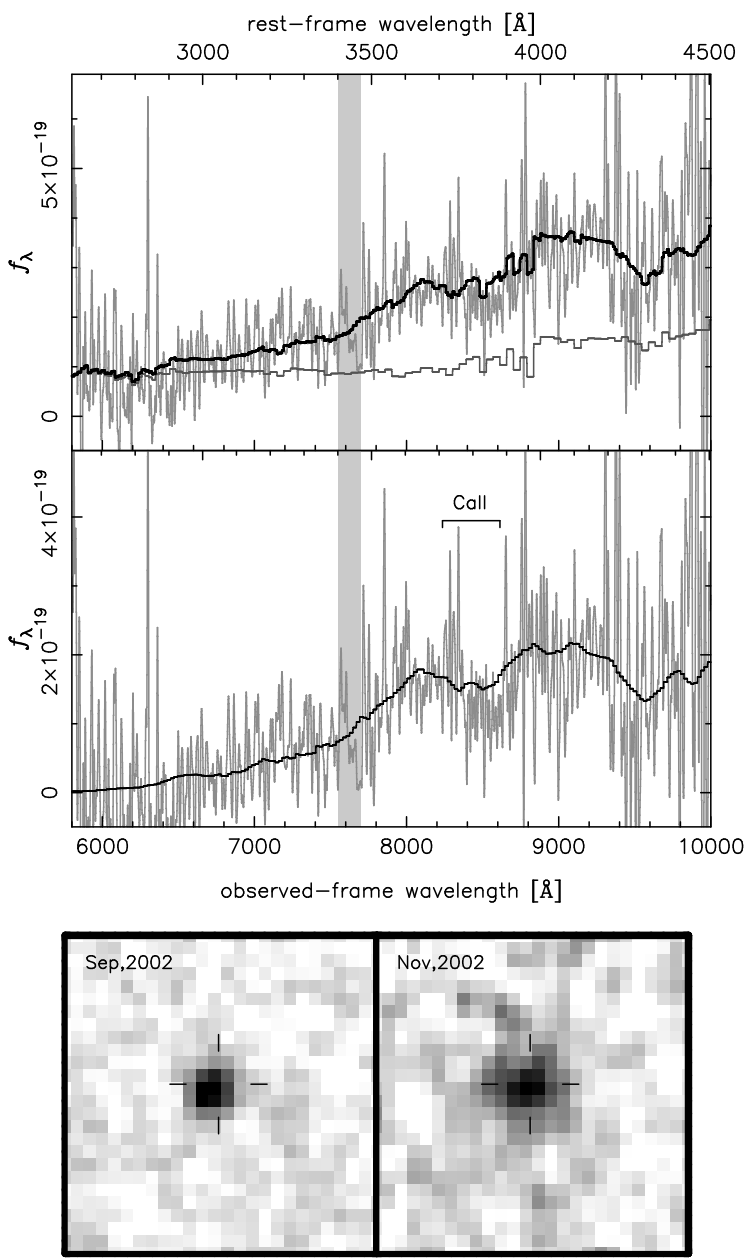

Fig. 9. (top): Spectra of SuF02-012 (SN 2002lc) at $z_{\mathrm{SN}}=1.22$ (gray) and $\mathrm{SN} 1991 \mathrm{~T}$ at $t=12$ days (black) redshifted to $z_{\mathrm{SN}}=1.22$. The C.I. is 2. (bottom): Finding charts of SuF02-012.

\subsection{Candidates from the HST Cluster Supernova Survey}

All the SN candidates shown in this section were found in the HST ACS survey for SNe Ia in distant galaxy clusters (HST Cluster Supernova Survey). On average, the SN candidates in this search are more distant than the ones found in earlier searches. Furthermore, since the survey aimed to find $\mathrm{SNe}$ Ia in early-type galaxies ${ }^{4}$, the relative brightness of such SN with respect to the host is usually small. Consequently, typing of these candidates generally needs to rely on other methods, such as the properties of the host. The principle aim of the spectroscopy is to get the redshift of the host. Note that the finding charts have been made from images taken with ACS. They have

4 If a galaxy has no detectable star formation and has the morphology of an early type galaxy, then we use the term early-type to describe the galaxy. It is a definition that is commonly used throughout the literature. To our knowledge, there has never been a Type II Supernova in a galaxy where there was no detectable signs of star formation. Of course, some star formation may be beyond detection in our optical spectra because it is either heavily obscured or because the star formation rate is very low. 

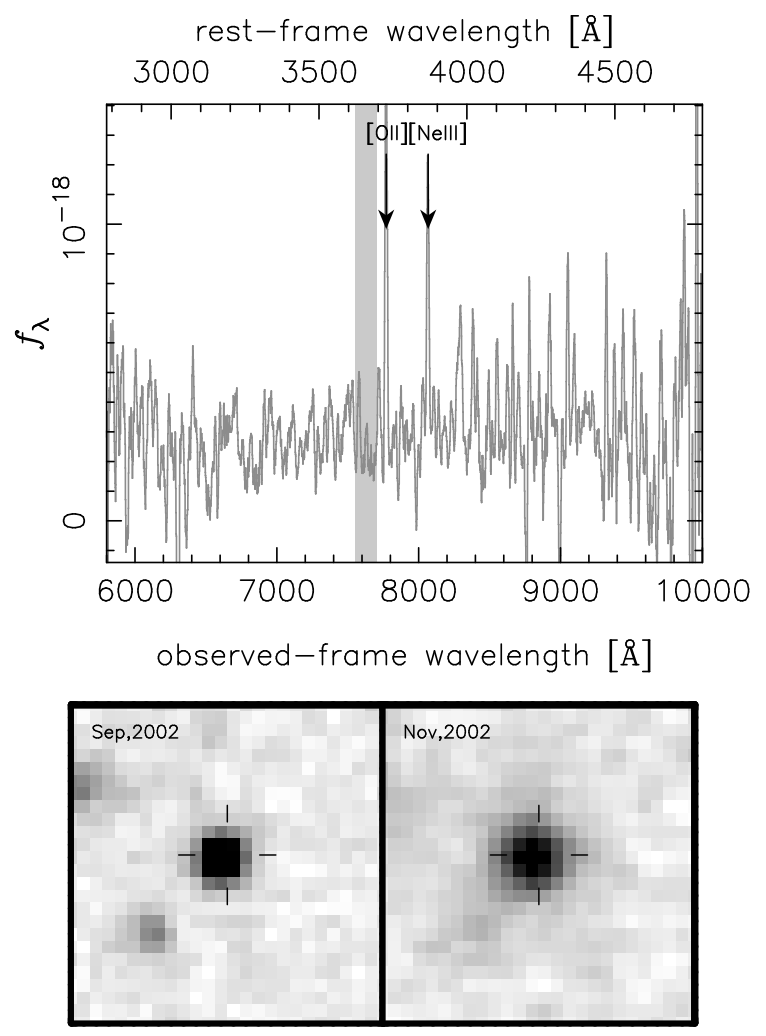

Fig. 10. (top): Spectra of SuF02-061 at $z_{\text {gal }}=1.085$. The C.I. is 1. (bottom): Finding charts of SuF02-061.

greater spatial resolution than those made from images taken with Suprime-Cam, which are smoothed by the seeing.

\subsection{1. $S C P 06 B 3$}

The spectrum of SCP06B3 is shown in Figure 11. It was taken on August 22, 2006 and has a total integration time of 7200 seconds. A second candidate, SCP06B4 (§3.3.2), was observed in the same slitmask. SCP06B3 appears near to a very diffuse galaxy that has a redshift of $z_{\text {gal }}=0.744$ from [O II], $\mathrm{H} \beta$, and [O III] emission lines. The spectrum is dominated by host galaxy light and a convincing type could not be obtained. The C.I. is set to 2 .

\subsection{2. $S C P 06 B 4$}

The spectrum of SCP06B4 is shown in Figure 12. It was taken on August 22, 2006 and has a a total integration time of 7200 seconds. SCP06B4 appears near to a diffuse galaxy that has a redshift of $z_{\text {gal }}=1.117$ as measured from [O II] and [NeIII] emission lines. The spectrum of SCP06B4 appears to be dominated by light from the host. A convincing type for the transient could not be derived, so the C.I. is set to 2.

\subsubsection{SCP06F6}

The extraordinary transient SCP06F6 was found on February 21, 2006. It shows a large (from below the detection limit in the ACS $i_{775}$ and $z_{850}$ bands) and slow $(\sim 5$ months in the observer frame) increase in flux (Dawson et al. 2006). For this interesting and unusual object, we took FOCAS spectra three times on April 222006 (6000 sec),
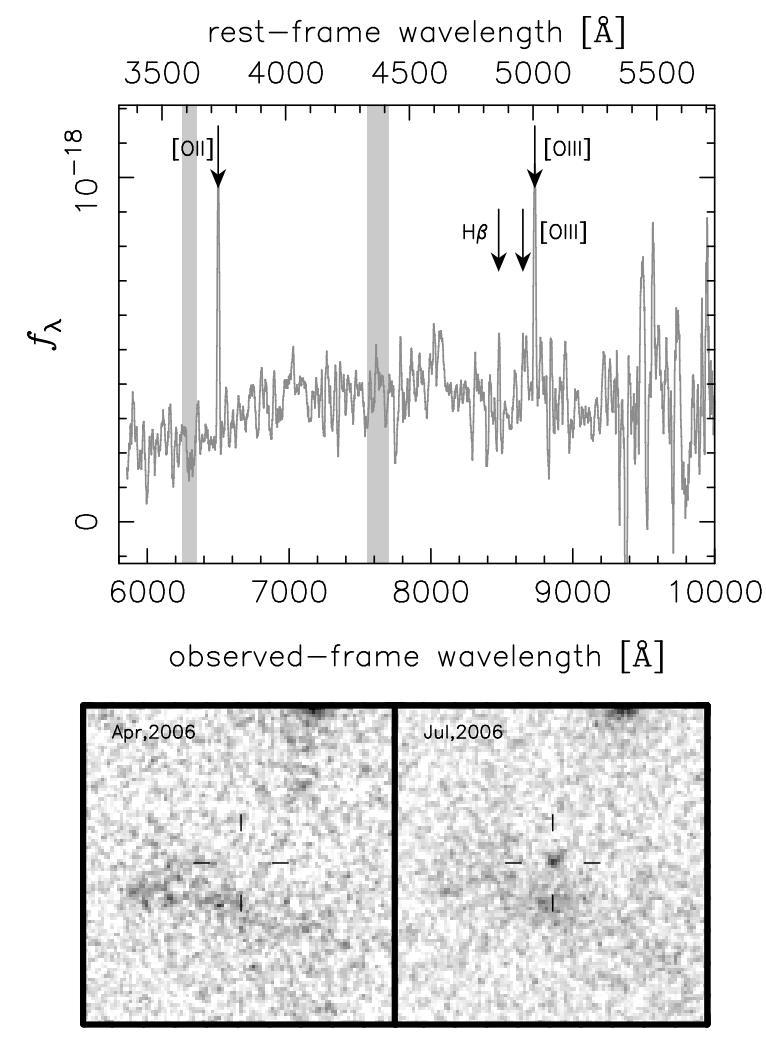

Fig. 11. (Top): Spectrum of SCP06B3 at $z_{\text {gal }}=0.744$ (gray). The C.I. is 2. (Bottom): Finding charts of SCP06B3. The size is $5^{\prime \prime} \times 5^{\prime \prime}$. North is up and east is left.

June 282006 (4800 sec), and August 222006 (2400 sec). However, the signal-to-noise ratio of the last two spectra are too low to see any spectral features. SCP06F6 seems to be hostless. The properties of this object are discussed in detail in Barbary et al. 2009. It is quite unlike a SN Ia, so the C.I. is set to 0.

\subsubsection{SCP06G3}

The spectrum of SCP06G3 is shown in Figure 13. It was taken on April 23 and 24, 2006 and has a total exposure time of 22,800 seconds. A second candidate, SCP06G4 (§3.3.5), was observed in the same slitmask. SCP06G3 appears to be associated with a galaxy that can be seen to the south of SCP06G3 (see Figure 13). A single strong emission line, which we identify as [O II] from the host galaxy, leads to a redshift of $z_{\text {gal }}=0.962$. Weak Balmer lines and a weak $4000 \AA$ break are also detected. The position angle of this slitmask was optimized for SCP06G4, which meant that the slit could not be set along the line joining SGP06G4 and the center of the host galaxy. Also, the spectrum was taken 24 days after maximum light, so it consists mostly of light from the host galaxy. For these reasons, this candidate could not be typed and a C.I. was not assigned.

\subsubsection{SCP06G4}

The spectrum of SCP06G4 is shown in Figure 14. It was taken on April 22 and 23, 2006 and the total integration was 22800 seconds. SCP06G4 is well separated from the putative host galaxy which lies to the northeast 

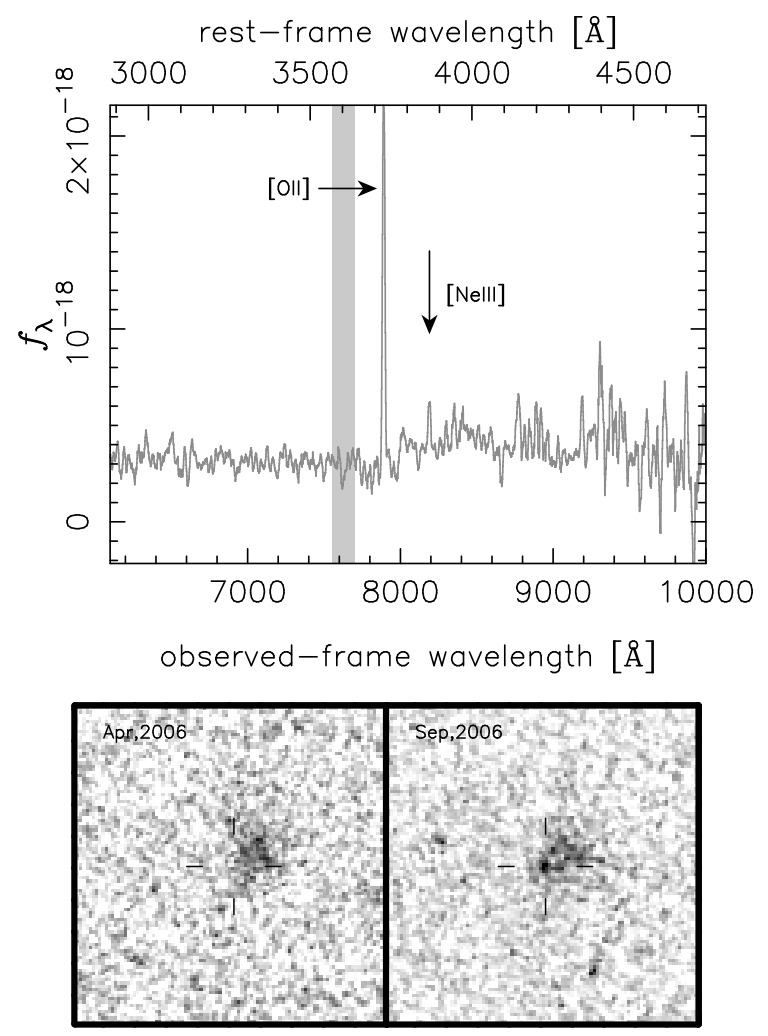

Fig. 12. (Top): Spectrum of SCP06B4 at $z_{\text {gal }}=1.117$ (gray). The C.I. is 2. (Bottom): Finding charts of SCP06B4.

of SCP06G4. The redshift of the host is $z_{\text {gal }} \sim 1.35$ from $\mathrm{CaII} \mathrm{H}$ and $\mathrm{K}$ absorption lines and the $4000 \AA$ break. A spectral fit to the host galaxy results in a similar redshift, $z_{\text {gal }}=1.350($ see $\S 4)$.

Although SCP06G4 is well separated from the host galaxy in the ACS images, the SN spectrum was strongly contaminated by light from the host galaxy. Thus, we subtract a spectrum that was extracted from a location that is at the same distance from the galaxy center to the SN but on the other side of the galaxy. The host subtracted spectrum of SCP06G4 is very noisy and can be fitted with a $\mathrm{SN} \mathrm{Ib/c} \mathrm{template} \mathrm{at} t=-6$ days redshifted to $z_{\mathrm{SN}}=1.36$ or the Hsiao Ia template at $t=-1$ days redshifted to $z_{\mathrm{SN}}=1.35$. Host galaxy subtraction using the BC03 templates also gives the best fitting result of $t=-1$ days redshifted to $z_{\mathrm{SN}}=1.35$ (the upper panel of Figure 14). The light curve is consistent with that of a SN Ia and $t_{\mathrm{sp}}$ matches $t_{\mathrm{LC}}$. The C.I. is 3 . The classification is supported by the characteristics of the host galaxy. The morphology of host is consistent with it being an elliptical galaxy (Meyers el al. in preparation) and no [O II] emission is detected in the spectrum of the host.

SCP06G4 is the most distant spectroscopically confirmed SN Ia in our sample and the most distant spectroscopically confirmed SN Ia using a ground based telescope. The confidence in the classification is similar to that that can be obtained for other similarly distant SNe that have ACS grism spectra (Riess et al. 2007). Clearly, in some
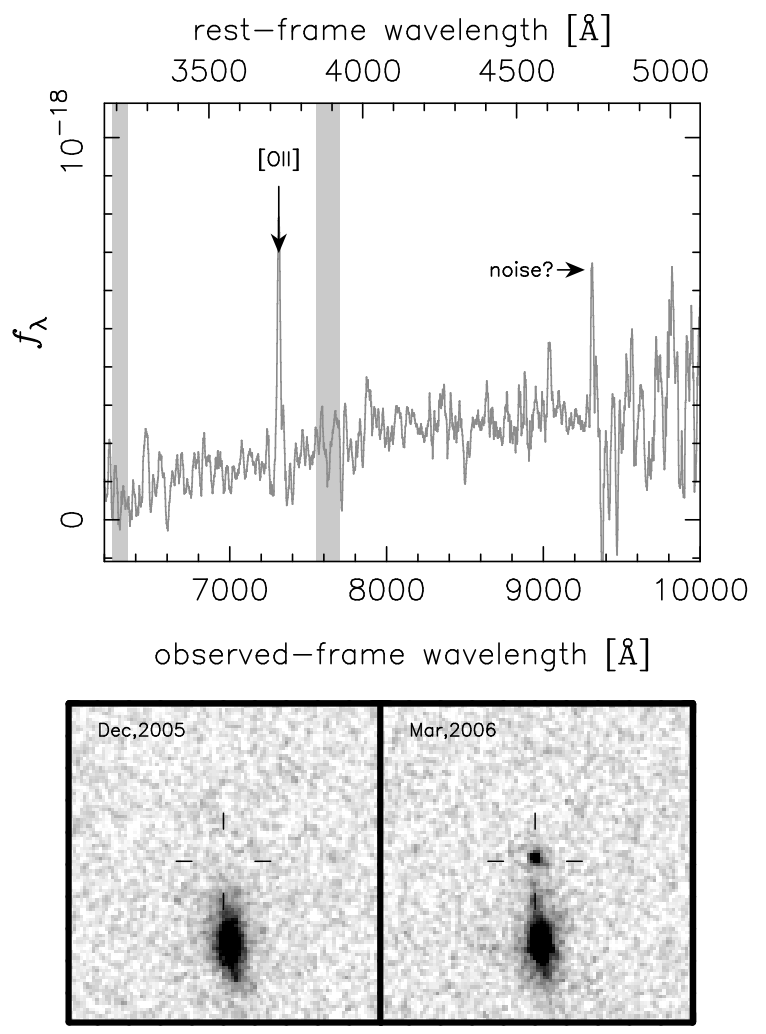

Fig. 13. (top): Spectrum of SCP06G3 at $z_{\text {gal }}=0.962$ (gray). The sharp feature at $9300 \AA$ is a noise artifact from the processing of the data and is not real. The C.I. is 2. (bottom): Finding charts of SCP06G3.

cases, one can do as well from the ground as from space, if atmospheric conditions are optimal, the host either faint enough or far enough away from the SN, integrations are long, and some care in adopting the optimal observing and data reduction strategies are employed.

3.3.6. $\mathrm{SCP06Н3}$

The spectrum of $\mathrm{SCP} 06 \mathrm{H} 3$ is shown in Figure 15. It was taken on April 22, 2006 and the total integration time was 6000 seconds. SCP06H3 appears to be associated with a very diffuse galaxy. The redshift of the galaxy is $z_{\text {gal }}=0.851$ as measured from [O II] and [O III]. The spectrum of SCP06H3 is well fitted to the Hsiao SN Ia template at $t=2$ days redshifted to $z_{\mathrm{SN}}=0.84$. The light curve is consistent with that of a SN Ia and $t_{\mathrm{sp}}$ matches $t_{\mathrm{LC}}$, so the C.I. is set to 4 .

3.3.7. SCP06N32

The spectrum of SCP06N32 is shown in Figure 16. It was taken on August 22, 2006 and the total integration time was 7200 seconds. SCP06N32 appears to be associated with a very diffuse and faint galaxy. We did not detect any spectral features from the galaxy. Although broad features are visible, the spectrum is noisy and the fitting results are inconclusive. The C.I. is set to 2 .

3.3.8. $S C P 05 P 1$

The spectrum of SCP05P1 is shown in Figure 17. It was taken on September 25, 2005 and the exposure time was 5400 seconds. SCP05P1 appears near to the center of 

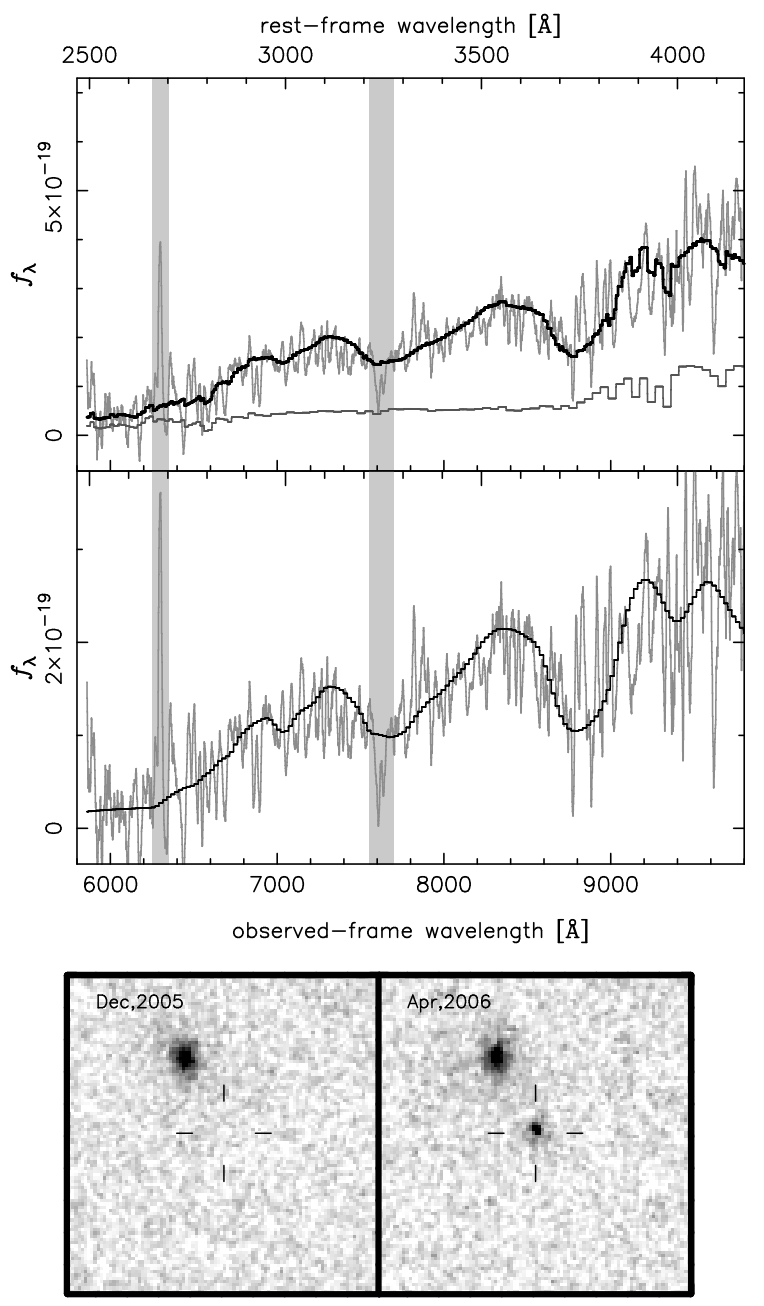

Fig. 14. (top): The spectrum of SCP06G4 at $z_{\text {gal,fit }}=1.350$ (gray) and the Hsiao Ia template at $t=-1$ days redshifted to $z_{\mathrm{SN}}=1.35$ (black). The C.I. is 3. (bottom): Finding charts of SCP06G4.

a very diffuse galaxy, which has a redshift of $z_{\text {gal }}=0.926$ from a $[\mathrm{O}$ II $]$ emission line. Since the spectrum was taken $t \sim 15$ days after the light curve peak, we restrict the spectral fits to late $\mathrm{SN}$ epochs. The best fit spectral template is SN 1998S, a Type IIn SN, at $t=27$ days redshifted to $z_{\mathrm{SN}}=0.91$, but the fit is poor. Allowing for some host galaxy light in the spectrum did not lead to a more conclusive fit. Since the spectrum was taken more than two weeks after maximum light, the confidence index is left unassigned.

\subsection{9. $S C P 05 P 9$}

The spectrum of SCP05P9 is shown in Figure 18. It was taken on October 25, 2005 and the exposure time was 12600 seconds. SCP05P9 is well separated from a galaxy that has redshift of $z_{\text {gal }}=0.821$ as measured from a [O II] emission line. The host galaxy is located to the northwest of SCP05P9 in the finding chart of Figure 18. The separation of the $\mathrm{SN}$ component from the host galaxy center is about 1 arcsec and, as in SCP06G4, we subtracted the host galaxy component from the spectrum at the SN po-
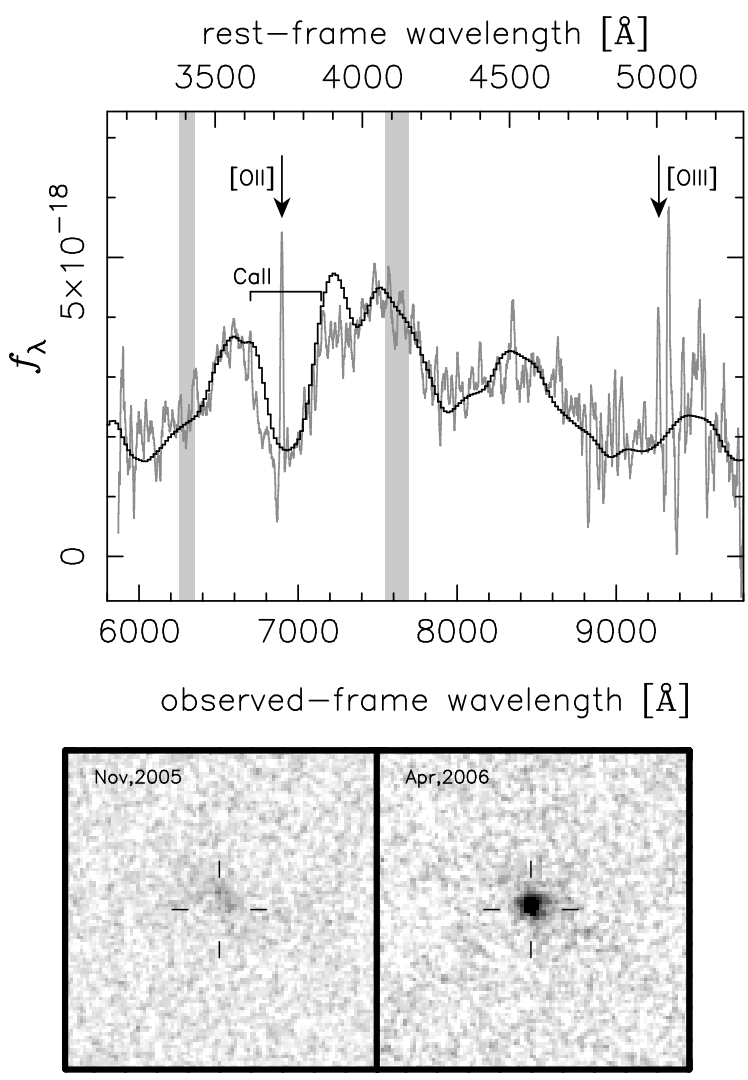

Fig. 15. (top): The spectrum of SCP06H3 at $z_{\text {gal }}=0.851$ (gray) and the Hsiao SN Ia template at $t=2$ days redshifted to $z_{\mathrm{SN}}=0.84$ (black). The C.I. is 4. (bottom): Finding charts of SCP06H3.

sition (§3.3.5). The best fit template over the observed wavelength range is the Hsiao Ia template at $t=-2$ days at $z_{\mathrm{SN}}=0.81$. The light curve is consistent with that of a $\mathrm{SN}$ Ia and $t_{\mathrm{LC}}$ matches $t_{\mathrm{SN}}$. The C.I. is 3 .

\section{Host Galaxy Spectral Fitting for SN Candidates in HST Cluster Supernova Survey}

As an alternative to spectroscopy, classification of the SN from other observables such as the lightcurve or the properties of the host can, and sometimes must, be employed. Identifying the host of a $\mathrm{SN}$ as an early type galaxy is a reasonable indication that the $\mathrm{SN}$ is a $\mathrm{SN} \mathrm{Ia}$, as SNe of other types are unlikely to occur in such galaxies. We fitted BC03 models to the hosts of five SN candidates in HST Cluster Supernova Survey to constrain the spectral properties of the hosts. The parameters of the fit were metallicity, star formation history (single burst or exponentially declining), star formation time scale $\tau_{\text {sf }}$, stellar age $t_{\text {age }}$, and extinction. In the fitting, we mask the region covering the atmospheric A-band around $7600 \AA$ and any possible emission lines since the BC03 models do not reproduce non-stellar spectral features. The spectral fitting also allowed a more accurate estimate of the redshifts of those host galaxies for which the Ca II H and K absorption 

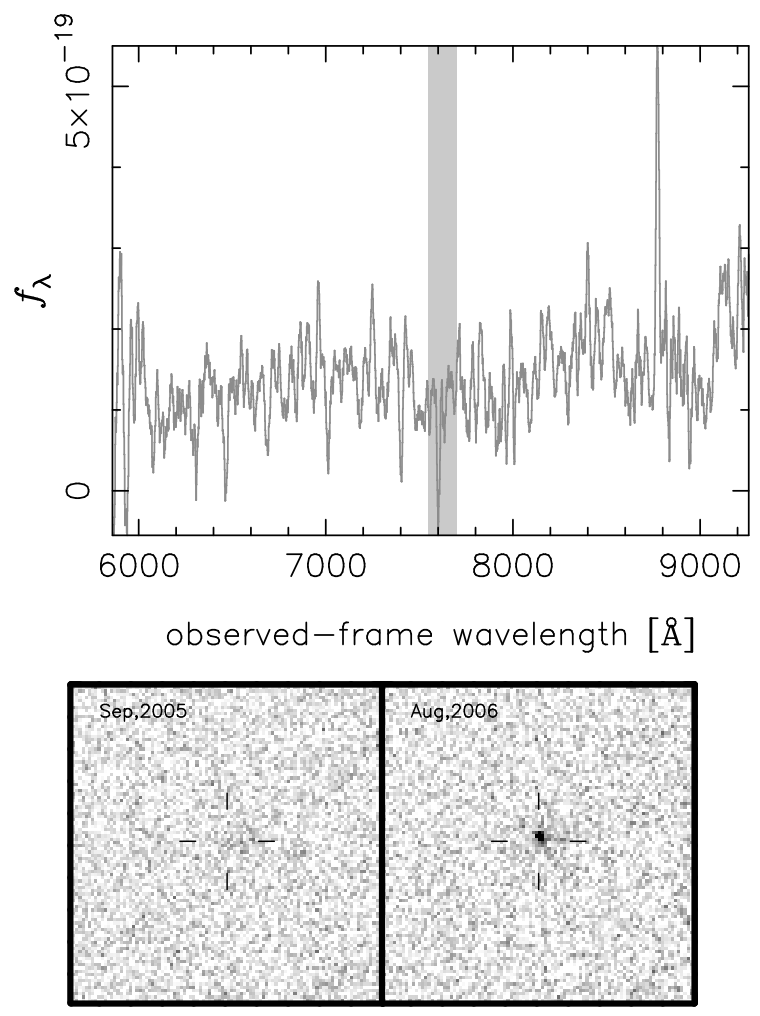

Fig. 16. (top): Spectrum of SCP06N32. The C.I. is 2. (bottom): Finding charts of SCP06N32.
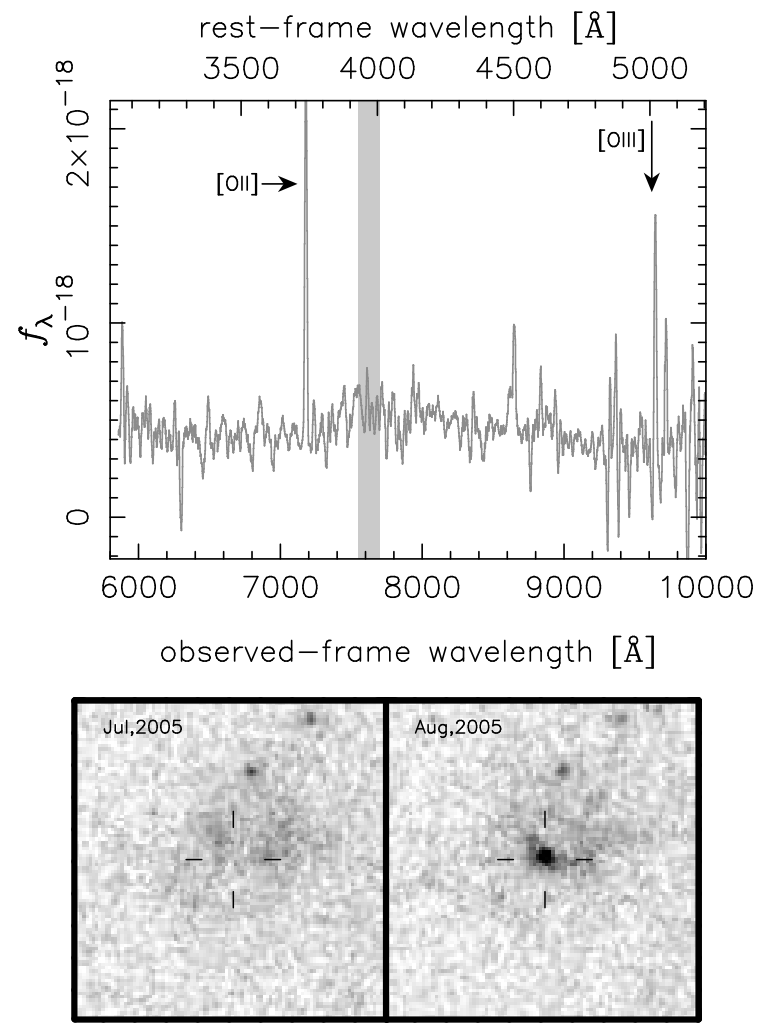

Fig. 17. (top): Spectrum of SCP05P1 at $z_{\text {gal }}=0.926$ (gray). The C.I. is 2. (bottom): Finding charts of SCP05P1.
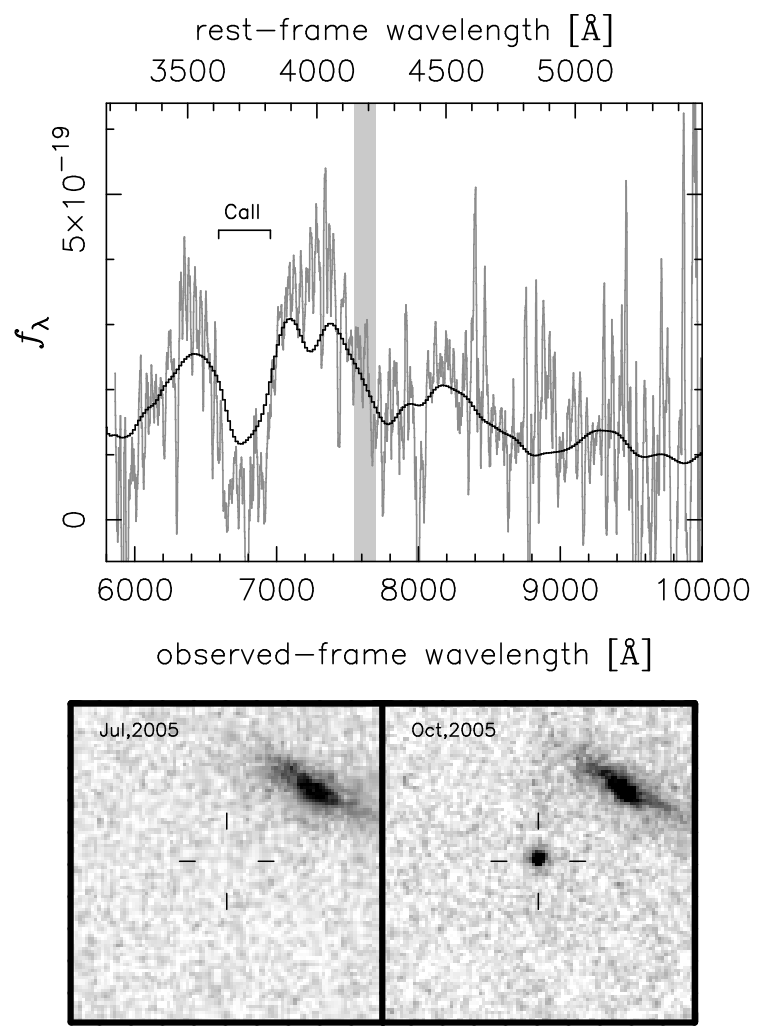

Fig. 18. (top): The spectrum of SCP05P9 at $z_{\text {gal }}=0.821$ (gray) and the Hsiao Ia template at $t=-2$ days redshifted to $z_{\mathrm{SN}}=0.81$ (black). We note that we determine the redshift from the spectrum of the host galaxy center and the [O II] emission line can not be seen in the SN spectrum figure. The C.I. is 3. (bottom): Finding charts of SCP05P9.

lines were detected with low signal-to-noise ratio.

The FOCAS spectra of the hosts of SCP05D0, SCP05D6, SCP06G4, SCP06K0, and SCP06K18 and the best fitting galaxy spectra are shown in Figures 19-23. We note that SCP06G4 is spectroscopically identified as a SN Ia* (§3.3.5). Observations for these host galaxies are summarized in Tables 2 and 3. In general, the contribution from the $\mathrm{SN}$ in these spectra can be ignored. SCP05D0, SCP05D6, SCP06K0, SCP06K18 were all reobserved when the contribution from the SN was negligible. For SCP06G4, a spectrum located at the center of the host was extracted. While some SN light may have leaked into the slit for these two objects, the contribution was estimated to be negligible for SCP06G4.

All of the observed galaxy spectra are well fitted with the models from $\mathrm{BC} 03$. Using the constraint that the age of the universe at the redshifts the galaxies were observed (for example, $6.0 \mathrm{Gyr}$ at $z \sim 1.0$ and $4.7 \mathrm{Gyr}$ at $z \sim 1.4$ ) should be larger than the respective ages of these galaxies, we obtain ranges (among the 40 best fitted templates) for the stellar age $t_{\text {age }}$ and the star formation time scale $\tau_{\text {sf }}$ for each galaxy. The uncertainties are large because the observed spectra are noisy. The 40 best fitted templates provide roughly the same $\chi^{2}$ values. The stellar ages $t_{\text {age }}$ are larger than the timescales of star formation $\tau_{\mathrm{sf}}$ for all 
galaxies. Among the well-fitted templates, templates with older ages generally have smaller extinction than those of younger ages. From our spectral fitting, we cannot exclude the possibility of significant extinction, but all the spectra are consistent with early-type galaxies with little extinction. The results above are summarized in Table 4.

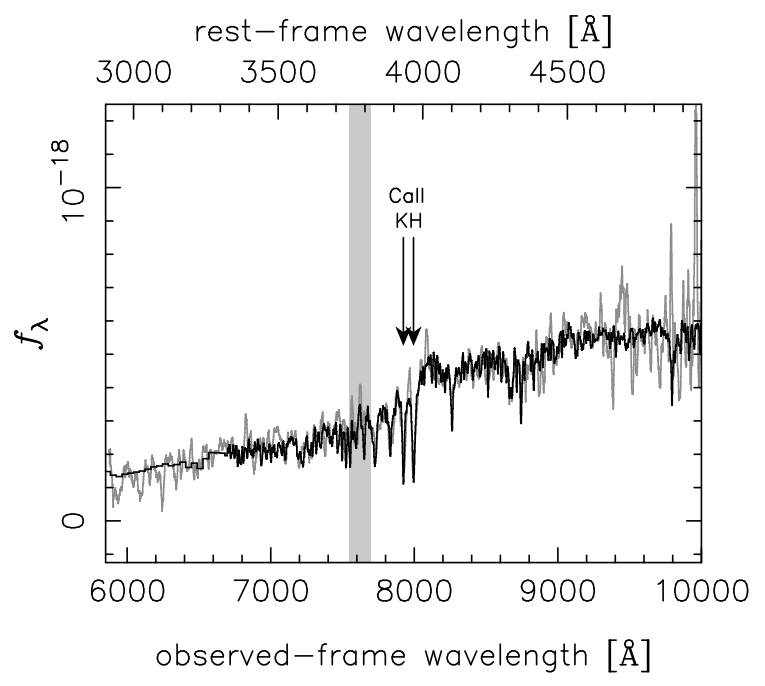

Fig. 19. Spectrum of the host of SCP05D0 (gray) and the best fitting galaxy template at $z_{\text {gal,fit }}=1.015$ (black).

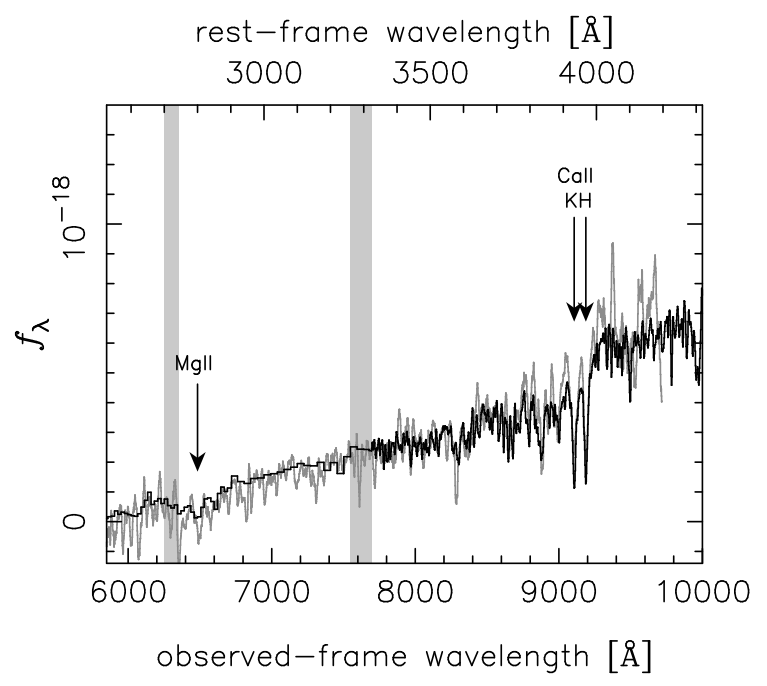

Fig. 20. Spectrum of the host of SCP05D6 (gray) and the best fitting galaxy template at $z_{\text {gal,fit }}=1.316$ (black).

\section{Classifying High Redshift Candidates}

The chance that any given high redshift candidate is positively identified as a SN of some type depends on many factors. A non-exhaustive list includes factors like the redshift of the candidate, the phase at which it was observed, the contrast with respect to the host, the wavelength coverage and signal-to-noise ratio of the spectrum, and, if

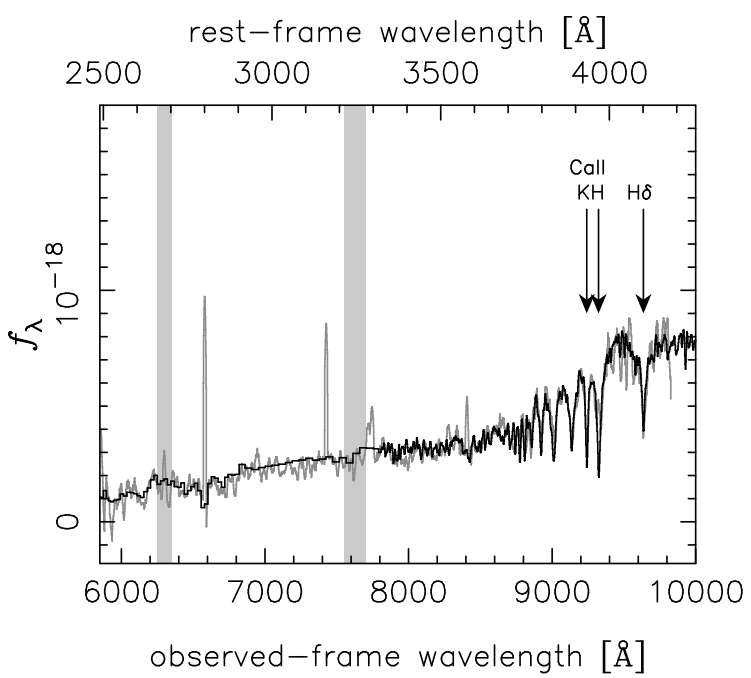

Fig. 21. Spectrum of the host of SCP06G4 (gray) and the best fitting galaxy template at $z_{\text {gal,fit }}=1.350$ (black).

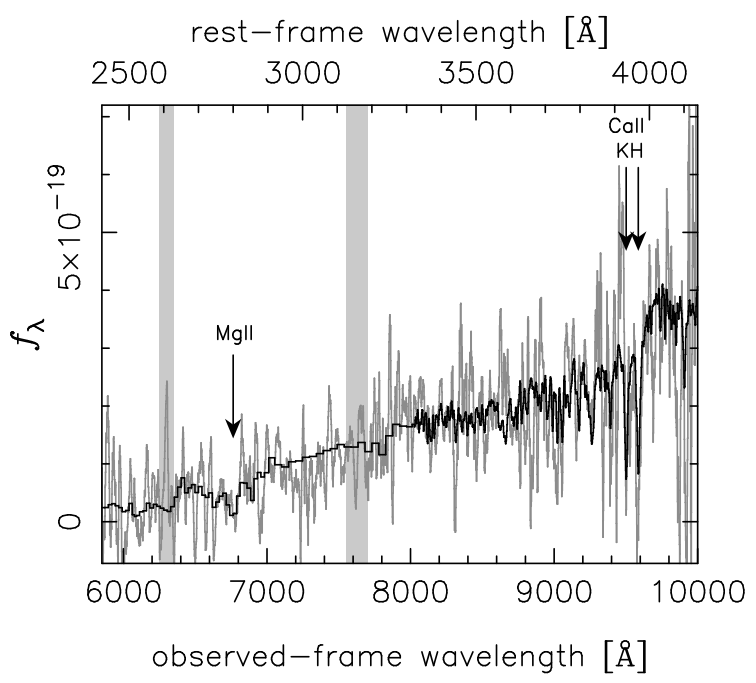

Fig. 22. Spectrum of the host of SCP06K0 (gray) and the best fitting galaxy template at $z_{\text {gal,fit }}=1.416$ (black).

photometry is used as part of the classification, the quality and amount of photometry. The lack of a large sample of UV spectra for SNe of all types, especially for CC SNe, is also a factor.

In Figures 24 and 25 we compare magnitudes, redshifts and signal-to-noise ratios of candidates that were successfully identified with those that were not. Only candidates that were observed within about two rest frame weeks of maximum light are included in these plots. The signal-tonoise ratios include both $\mathrm{SN}$ and host galaxy components except for SCP06G4 and SCP05P9, for which we subtract the spectrum of the host. Although exposure times and weather conditions differ from one candidate to the next, one generally finds, not surprisingly, that it is easier to identify the SN type when they are at lower redshift, when they are brighter and when the signal-to-noise of the 
Table 4. Spectral fitting results for SN hosts. The SN names are denoted in column $1 . \quad$ Columns 2 and 3 contain the redshifts measured from individual spectral features, some of which are not very precise due to the noisy nature of the spectra, and the redshifts derived from the spectral fitting, respectively. Columns 4 and 5 contain the fitted ranges of ages and star formation timescales. Both are in Gyr. In columns 6 and 7 , the qualitative strengths of the CaII H and $\mathrm{K}$ absorption lines and the $4000 \AA$ break are shown, respectively.

\begin{tabular}{crccccc}
\hline SN & $z_{\text {gal }}$ & $z_{\text {gal,fit }}$ & $t_{\text {age }}[\mathrm{Gyr}]$ & $\tau_{\text {sf }}[\mathrm{Gyr}]$ & Ca II H and K & $4000 \AA$ break \\
\hline SCP05D0 & 1.015 & 1.015 & $1.0-3.0$ & $0.0-0.5$ & clear & clear \\
SCP05D6 & 1.315 & 1.316 & $1.5-3.0$ & $0.0-0.1$ & clear & clear \\
SCP06G4 & $\sim 1.35$ & 1.350 & $1.0-1.5$ & $0.0-0.3$ & exist & clear \\
SCP06K0 & $\sim 1.41$ & 1.416 & $1.5-3.0$ & $0.0-0.3$ & exist & clear \\
SCP06K18 & $\sim 1.41$ & 1.411 & $1.5-3.0$ & $0.0-0.5$ & exist & clear \\
\hline
\end{tabular}

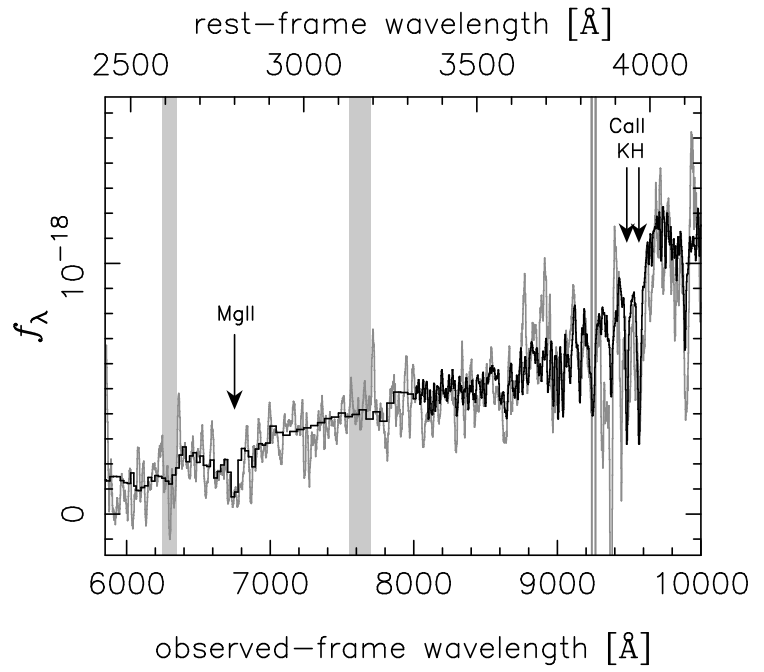

Fig. 23. Spectrum of the host of SCP06K18 (gray) and the best fitting galaxy template at $z_{\text {gal,fit }}=1.411$ (black).

spectrum is higher.

Also critical is the phase of the SN when the spectrum is taken. In Figure 26, $t_{\mathrm{LC}}$ (the light curve epoch at the time the spectrum was taken) is plotted as a function of redshift. Only objects with well-sampled light curves are shown, so objects like CL1604_0-1 and SDF1 are excluded. Black circles and boxes are SNe that have a C.I. that is greater than 2. Considering only the spectra within the shaded region, 5 out of 11 candidates observed with FOCAS are spectroscopically identified as SN Ia or SN Ia*. Of these 5, 4 are below $z=1.1$. To increase the number of candidates in this comparison, we add 8 SN candidates from the HST Cluster Supernova Survey (Dawson et al. 2009) that were observed with FORS2: SCP06A4, SCP06C0, SCP06C1, SCP05D0, SCP05D6, SCP06R12, SCP06U4 and SCP06Z5. Considering only the spectra within the shaded region and combining the results from FOCAS and FORS2, 9 out of 18 candidates $(50 \%)$ are spectroscopically identified as SN Ia or SN Ia*. Of these 9,8 are below $z=1.1$. FORS2 and FOCAS proved to be equally effective in this redshift interval.

Clearly, $z>1.1$ SNe Ia are more difficult to confirm spectroscopically with ground based facilities. This is not a surprising result. They are fainter and the peak of the flux is shifted into a wavelength region where the night sky is bright. However, it is possible to confirm SNe be-

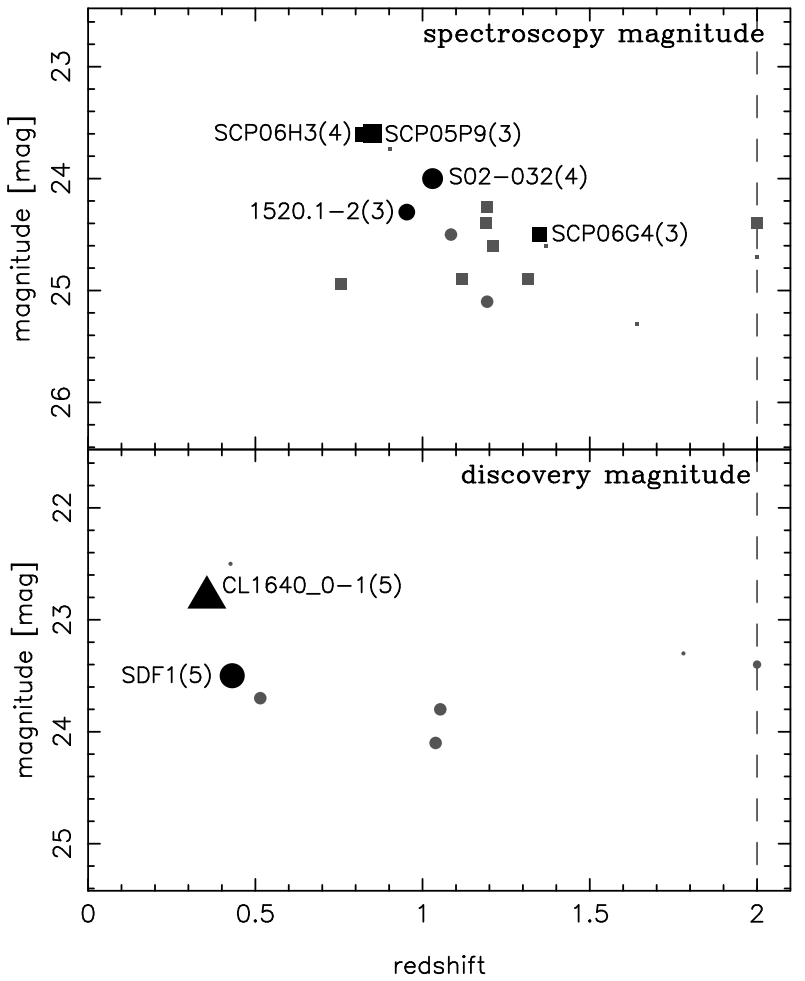

Fig. 24. The distribution of SN candidate magnitudes as a function of redshift. If a well-enough sampled lightcurve is available, the magnitude at the time the spectrum was taken is used and is shown in the upper panel. Otherwise the magnitude at the time of discovery is used and is shown in the lower panel. The symbol size is proportional to the degree of confidence that the candidate is a SN Ia. We plot candidates with C.I. $\leq 2$ in gray. Triangle, circles and boxes represent $R_{c}$, $i^{\prime}$, and $z^{\prime}$ - magnitudes, respectively. Symbols on the vertical gray dashed line at $z=2$ are objects whose redshifts can not be determined. 


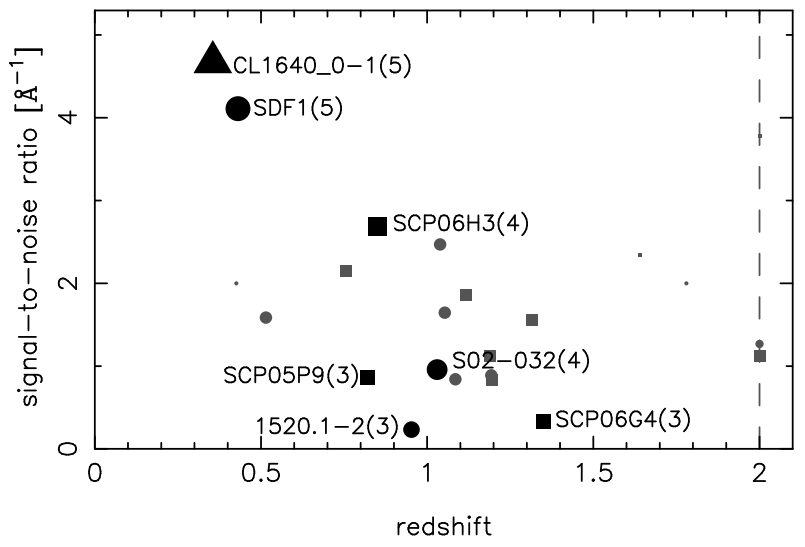

Fig. 25. The distribution of signal-to-noise ratios of the spectra as a function of redshift. Symbols are the same as those in Figure 24. For SCP05P9 and SCP06G4, we plot signal-to-noise ratios of host-subtracted SN component spectra. Symbols on the vertical gray dashed line at $z=2$ are objects whose redshifts can not be determined. For reference, a 2-hour, low resolution $(R \sim 500)$ spectrum with FOCAS of a $z^{\prime} \sim 25$ mag point source has $S / N=1\left[\AA^{-1}\right]$. The signalto-noise ratios are not scaled to a common exposure time. Doing so tends to stretch the plot vertically.

yond $z=1.1$ with optical instrumentation, if conditions are ideal and integrations are long. SCP06G4 in this paper is an example. With the upgrade of optical spectrographs with CCDs that have improved red sensitivity and minimal fringing (as was recently done for LRIS on Keck and is now being done for FOCAS on Subaru), it should be possible to increase the yield of spectroscopically classified SNe Ia with ground based facilities beyond $z=1.1$. Laser guide star adaptive optics (LGS-AO) assisted infra-red spectroscopy may be another alternative; however, only a few SNe Ia are expected to have a tip-tilt star that is both near enough and bright enough for LGSAO to be effective. SCP05D6, for example, was imaged in the $H$-band (rest frame $R$-band) with the LGS-AO system at the Keck Observatory (Melbourne et al. 2007).

The strategy adopted in the HST Cluster SN Survey is an alternative. Since Type II SN are extremely rare in early-type galaxies, spectroscopic confirmation of the SN type in such galaxies is unnecessary. Spectroscopy is still necessary to obtain redshifts. However, the amount of time needed to obtain redshifts is generally much less than the time needed to confirm the SN type, and the spectrum can be taken long after the $\mathrm{SN}$ is no longer is visible. In one case, SCP05D0, a spectrum of the host was available from archived data and was taken before the SN exploded (Dawson et al. 2009). The key here is to show that the amount of star formation in the host is low enough that $\mathrm{SN}$ of other types are unlikely.

\section{Summary}

We presented spectra of SN candidates obtained with FOCAS on the Subaru 8.2-m telescope. Seven active candidates were identified as SNe Ia, including SCP06G4 at

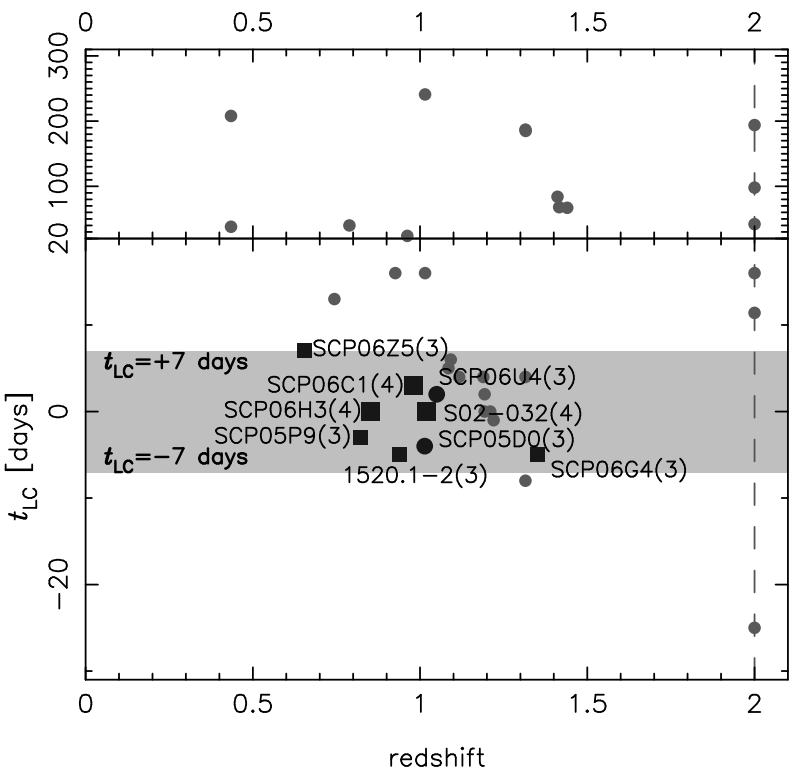

Fig. 26. The distribution of the epoch the spectra were taken as a function of redshift. We only consider objects with well-sampled light curves that have well a determined date of peak brightness. Circles indicate candidates that we could only identify as a SN Ia when a spectrum of the host was available. The gray region is $-7 \leq t_{\mathrm{LC}} \leq 7$ days. We note that circles at $z=2$ are objects without spectroscopic redshift determination and we plot observed-frame epochs for these objects. The object at $t_{\mathrm{LC}} \sim-25$ days is the extraordinary transient SCP06F6.

$z_{\mathrm{SN}}=1.35$, the most distant SN Ia to be spectroscopically identified with a ground-based telescope. Redshifts were obtained for all but 7 of the remaining 32 candidates based on the host galaxy spectra. An additional 4 candidates are identified as likely SNe Ia from the spectrophotometric properties of their hosts. The spectral properties of these hosts found in the HST Cluster Supernova Survey were examined by comparing their spectra with BC03 model spectra. All of the host galaxy spectra indicate that they are passively evolving galaxies that have quenched their star forming activities.

We also investigated the factors affecting the classification of SNe and found that it is critical to take spectra within a week of maximum light. This requires secure early detection, well-sampled light curves and prompt spectroscopic follow-up.

We thank the anonymous referee for providing helpful comments and suggestions. TM and YI are financially supported by the Japan Society for the Promotion of Science (JSPS) through the JSPS Research Fellowship. CL acknowledges the financial support from the Oskar Klein Centre at the University of Stockholm. This work was supported in part with scientific research grants (15204012 and 17104002) from the Ministry of Education, Science, Culture, and Sports of Japan, and a JSPS core-to-core program "International Research Network for Dark Energy". Financial support for this work was provided in part by NASA through program GO-10496 from 
the Space Telescope Science Institute, which is operated by AURA, Inc., under NASA contract NAS 5-26555. This work was also supported in part by the Director, Office of Science, Office of High Energy and Nuclear Physics, of the U.S. Department of Energy under Contract No. AC02-05CH11231. Part of the Suprime-Cam observations were done during the guaranteed time observation of Suprime-Cam and we thank for the Suprime-Cam instrument team. We also appreciate much help by the SDF and SXDS project team members. We also thank for Youichi Ohyama, who helped our observations as a support scientist of FOCAS. Data analysis were in part carried out on common use data analysis computer system at the Astronomy Data Center, ADC, of the National Astronomical Observatory of Japan.

\section{References}

Aldering, G., et al. 1998, IAU Circ. 7046, 1

Aldering, G., et al. 2002, SPIE, 4836, 61

Amanullah, R., et al. 2009, in preparation

Astier, P., et al. 2006, A\&A, 447, 31

Barbary, K., et al. 2009, ApJ, 690, 1358

Barris, B. J., et al. 2004, ApJ, 602, 571

Benítez, N. et al. 2003, RMxAC, 16, 39

Bohlin, R. C., Dickinson, M. E., \& Calzetti, D. 2001, AJ, 122, 2118

Borgne, D. L., et al. 2006, ApJ, 642, 48

Bruzual \& Charlot 2003, MNRAS, 344, 1000

Cardelli, J. A., Clayton, G. C., \& Mathis, J. S. 1989, ApJ, 345, 245

Coil, A. L. et al. 2000, ApJL, 544, L111

Cuillandre, J.-C., et al. 2000, SPIE, 4008, 1010

Dawson, K., et al. 2006, CBET, 546, 1

Dawson, K., et al. 2009, AJ, 138, 1271

Drake, A. J., et al, 2009, ApJ, 696, 870

Drake, A. J., et al, 2009, arXiv:0908.1990

Doi, M., et al. 2001, IAUC, 7649, 1

Doi, M., et al. 2003, IAUC, 8119, 1

Eisenhardt, P., et al. 2008, ApJS, 684, 905

Eisenstein, D. J., et al. 2005, ApJ, 633, 560

Frieman, J. A., et al. 2008, AJ, 135, 338

Hamuy, M., et al. 1996, AJ, 112, 2408

Hamuy, M., et al. 2006 PASP, 118, 2

Hicken, M., et al. 2009, arXiv:0901.4804

Hodapp, K. W., et al. 2003, PASP, 115, 1388

Hook, I. M., et al. 2004, PASP, 116, 425

Howell, D. A., et al. 2005, ApJ, 634, 1190

Hsiao, E. Y., et al. 2007, ApJ, 663, 1187

Ihara, Y., et al. 2009, in preparation

Iwamuro, F., Motohara, K., Maihara, T., Hata, R., \& Harashima, T. 2001, PASJ, 53, 355

Iye, M., et al. 2004, PASJ, 56, 381

Jha, S., et al. 2006, AJ, 131, 527

Kaiser, N., et al. 2002, SPIE, 4836, 154

Kashikawa, N., et al. 2002, PASJ, 54, 819

Kashikawa, N., et al. 2004, PASJ, 56, 1011

Keller, S. C., et al. 2007, PASA, 24, 1

Komatsu, E., et al. 2009, ApJS, 180, 330

Konishi, K., et al. 2009, in preparation

Law, N. M., et al. 2009, arXiv:0906.5350

Lidman, C., et al. 2005, A\&A, 430, 843
Lubin, L. M., Mulchaey, J. S., \& Postman, M., 2004, ApJL, 601, L9

Melbourne, J., et al. 2007, AJ, 133, 2709

Meyers, J., et al. 2009, in preparation

Miknaitis, G., et al. 2007, ApJ, 666, 674

Miyazaki, S., et al. 2002, PASJ, 54, 833

Moorwood, A. et al. 1998, The Messenger 94, 7

Morokuma, T., et al. 2008, ApJ, 676, 163

Motohara, K., et al. 2002, PASJ, 54, 315

Nugent, P., Kim, A., \& Perlmutter, S., et al. 2002, PASP, 114, 803

Oda, T., \& Totani, T. 2005, PASJ, 630, 59

Oda, T., et al. 2008, PASJ, 60, 169

Oke, J. B., et al. 1995, PASP, 107, $375 \mathrm{O}$

Patat, F., et al. 2001, ApJ, 555, 900

Perlmutter, S., et al. 1999, ApJ, 517, 565

Phillips, M. M. 1993, ApJL, 413, 105

Reindl, B., Tammann, G. A., Sandage, A., \& Saha, A. 2005, ApJ, 624, 532

Riess, A. G., et al. 1998, AJ, 116, 1009

Riess, A. G., et al. 1999, AJ, 117, 707

Riess, A. G., et al. 2007, ApJ, 659, 98

Riess, A. G., et al. 2004, ApJ, 607, 665

Sako, M., et al. 2008, AJ, 135, 348

Sekiguchi, K. (SXDS) 2004, AAS, 205, 8105

Sekiguchi, K., et al. 2009, in preparation

Sheinis, A. I., et al. 2002, PASP, 114, 851

Smith, N., et al. 2007, ApJ, 666, 1116

Spergel, D. N., et al. 2003, ApJS, 148, 175

Spergel, D. N., et al. 2007, ApJS, 170, 377

Stocke, J. T., et al. 1991, ApJS, 76, 813

Suzuki, N., et al. 2009, in preparation

Takanashi, N., Doi, M., \& Yasuda, N., 2008, MNRAS, 389, 1577

Tanaka, M., et al. 2008, A\&A, 489, 571

Thompson, R. I. et al. 1999, AJ, 117, 17

Tokita, K., 2009, Ph.D.Theis, Univ. of Tokyo

Tonry, J. L., et al. 2003, ApJ, 594, 1

Totani, T., et al. 2005, ApJL, 621, L9

Totani, T., Morokuma, T., Oda, T., Doi, M., \& Yasuda, N. 2008, PASJ, 60, 1327

Tripp, R., 1998, A\&A, 331, 815

Vikhlinin, A., et al. 2009, ApJ, 692, 1060

Wang, L., et al. 2003, ApJ, 590, 944

Wood-Vasey, W. M., et al. 2007, ApJ, 666, 694

Yasuda, N., et al. 2002, IAUC, 7971, 1

Yasuda, N., et al. 2003, AAS, 203, 8211

Yasuda, N., et al. 2009, in preparation

Zheng, C., et al. 2008, AJ, 135, 1766 